\title{
How does grazing incidence ultrasonic microscopy work? A study based on grain-scale numerical simulations *
}

\author{
Michał K. Kalkowski ${ }^{\mathrm{a}, *}$, Michael J. S. Lowe ${ }^{\mathrm{a}}$, Martin Barth ${ }^{\mathrm{b}}$, Marek Rjelka ${ }^{\mathrm{b}}$, Bernd Köhler ${ }^{\mathrm{b}}$ \\ ${ }^{a}$ Mechanical Engineering, Imperial College London, SW7 2AZ, UK \\ ${ }^{b}$ Fraunhofer Institute for Ceramic Technologies and Systems IKTS, 01109 Dresden, Germany
}

\begin{abstract}
Grazing incidence ultrasonic microscopy (GIUM) is an experimental method for visualising the microstructures of polycrystals with local preferential orientations. It has previously been demonstrated on an austenitic stainless steel weld exposing grains much smaller than the propagating wavelength, but the physical mechanism of the method has only been proposed as a hypothesis. In this paper, we use grain-scale finite element simulations based on the EBSD measurements to verify the principles behind GIUM images further and to assess how deep does the method penetrate the component under examination. The simulations indicate that while lateral contraction of grains contains microstructure signatures, the free surface effect is the crucial factor contributing to the generation of the images. Further, we show that only features up to the depth in the order of the average grain size in that direction can be visualised.
\end{abstract}

\section{Introduction}

The microstructure plays a vital role in material science. The grain size and crystallite type are essential 3 parameters for polycrystals and alloys. In polycrystalline metals, the grain orientation often varies with 4 position and determines the material properties. Prominent examples are austenitic and dissimilar welds in is polishing and etching, producing contrast in the succeeding optical imaging by different etch rates for

\footnotetext{
${ }^{\star}$ This manuscript version is made available under the CC-BY-NC-ND 4.0 license

* Corresponding author

Email address: m.kalkowski@imperial.ac.uk (Michał K. Kalkowski) 
different crystallographic orientations. A more detailed orientation map can be determined by exploiting the interference of scattered X-rays or electron beams. While the X-ray Laue interference method is costly, the Electron Back-Scatter Diffraction (EBSD) [1] is the quasi-gold standard for this type of examination.

As the elastic properties in grains are orientation-dependent, measurements with elastic waves have the potential not only to visualise the shapes of the grains, but also to determine the crystallographic orientations. Indeed, the visualisation of grains was one of the first applications of the Scanning Acoustic Microscope (SAM) 2]. The use of cylindrical lenses in connection with a special measurement procedure called 'V( $\mathrm{z}$ ) curve determination' allowed, after calibration, a precise, orientation-dependent determination of the leaky surface acoustic wave velocity (LSAW) [3]. It was verified that this orientation distribution of the LSAW velocity can be inverted to obtain the crystallographic orientation [4. However, this was demonstrated only for a given point (and therefore one grain) and, as far as we know, due to the slowness of the measurements, it could not be extended to give orientation maps of larger areas.

Another method related to scanning acoustic microscopy is called the Atomic Force Acoustic Microscopy (AFAM) [5]. In this method, the elastic properties are sensed by the dynamic interaction of a mechanical tip with the surface. The spatial resolution of AFAM is determined by the contact area, which can be much smaller than the acoustic wavelength. Therefore, the grain structure can be mapped at a much higher resolution [6, 7] compared to SAM.

A very attractive alternative to obtain grain structure information by acoustic waves is called spatially resolved acoustic spectroscopy (SRAS) [8-11. Thereby a laser beam structured as a fringe grating excites Rayleigh waves which are picked up optically in the vicinity. In the first version of the method, the laser beam emits a burst of single pulses with a frequency of $82 \mathrm{MHz}$. The fringe spacing can be changed from burst to burst with a repetition rate of $1 \mathrm{kHz}$. With fixed wave frequency $f$, the fringe distance $d$ matching the wavelength $\lambda$ at $f$ excites the wave most efficiently. Thus, the velocity can be obtained as a maximum in the signal amplitude over $d$ by calculating $c=f d$ [8, 9]. In the second variant of SRAS, the grating distance $\mathrm{d}$ is kept constant, and the laser excites a pulse, so the frequency peak of the spectrum of the surface vibration measured nearby gives the 'local' surface velocity by the same formulae [10. The second variant is faster than the first one because the sweep of the grating distance $d$ is not necessary, and the spectrum of the detected signal is obtained by FFT nearly instantaneously. Both variants of SRAS are much faster than the $V(z)$ procedure of SAM which involves a mechanical $z$-scanning at each point in addition to the rotation of the cylinder lens. Therefore, with SRAS, it is possible to determine the Rayleigh wave speed over a grid of scan points for several orientations, which is enough to produce a grain orientation map. The spatial resolution of this method is a couple of Rayleigh wavelengths, and as such comparable with the resolution of SAM, but it cannot reach the resolution of EBSD. A thorough overview of SRAS with additional literature is given in [1].

Motivated by the challenges in ultrasonic testing of austenitic and dissimilar welds, there was extensive 
work on visualisation of ultrasonic wave propagation over cross-sections using scanning laser vibrometry 12 14, and other methods, such as e.g. electrodynamic probes [15. For laser vibrometry, a considerable improvement of the image quality was achieved by advanced averaging algorithms [13, 16]. Owing to that, grain structures could be glimpsed in the acquired wave propagation image sequences. Surprisingly, even relatively simple data manipulations of the image stack produced visualisations that resembled the grain structure provided by metallography [17. In the experimental arrangement, the primary longitudinal wave was at grazing incidence to the measurement plane, so the approach was called 'grazing incidence ultrasonic microscopy' (GIUM). Improved evaluation algorithms were subsequently developed, and the findings were verified by application to dissimilar welds with buffering [18, by using different incidence angles of the primary wave [19] and by comparison of the GIUM results to an EBSD map [20].

There has been some speculation about the GIUM contrast mechanism [17, but up to now, there was no confirmation whether the common assumption was correct. With the suggested mechanism, the method is a kind of nearfield method, implying the resolution is in principle limited only by the size of the detection area: the laser spot size. This would be a potential advantage compared to SRAS, giving us a strong motivation to pursue a deeper insight into the contrast mechanism of GIUM.

Finite element simulations are a versatile high-fidelity tool, which can replace or supplement experiments. They become particularly advantageous when the measurements are costly, time consuming or affected by restricted access. Recent advances in the use of computational resources, by e.g. moving the calculations to the GPU [21] enabled for retaining fine scale details in the model. Over the past years, grain-scale finite element simulations have become an increasingly popular tool for modelling wave propagation in polycrystals. Of interest here are both investigations into the fundamental phenomena, such as attenuation [22 26] and backscatter [27, and application-oriented solutions, such as those dedicated to austenitic steel welds [28, 29]. The numerical capacity also allowed incorporating highly-detailed material examinations into FE modelling of ultrasound [14, 30, which will be further developed in this paper.

We propose to use grain-scale finite element simulations based on the EBSD data and synthetic microstructures to further investigate the physical principle behind GIUM and assess its penetration depth. The study consists of both two- and three-dimensional simulations (2D and 3D). The motivation for using 2D simulations to represent an essentially 3D setup is twofold. First, the weld is characterised only over the EBSD measurement plane, and no information about the microstructure underneath the surface is available. Second, 2D finite element models are considerably smaller in size and require less computational resources to run. Finally, in austenitic stainless steel welds, grain orientations do not vary significantly along the out-of-plane direction.

The structure of this draft is the following. At the start, we outline the GIUM experimental setup, present the workflow for processing raw EBSD data and the process for generating GIUM images used throughout this work, and recall previously obtained experimental images. Then, we discuss the 2D grain- 
scale FE model and images obtained from the simulations (displacement, strains, and resultant out-of-plane stress). Following from that, we render the model three-dimensional by uniformly extruding the grains obtained from the reconstruction in the depth direction $(z)$. The 3D simulation outputs the out-of-plane displacement, allowing us to generate GIUM images in an analogous way to the experimental measurement. Having reported EBSD-based simulations, we turn our attention to the fundamental phenomena in the vicinity of the free surface, to identify the most plausible mechanism that makes the imaging effective. Finally, we place a randomly generated polycrystal with uniformly distributed grain sizes behind the weld to assess how deep does the method penetrate.

\section{Preliminaries}

\subsection{The sample and the experimental arrangement}

In this paper, we use a sample produced during a previous project on the improvement of ultrasonic inspectability of coarse-grained austenitic and dissimilar welds [31. The welding was performed on $16 \mathrm{~mm}$ thick plates of 1.4432 (X2CrNiMo17-12-2 or 316L) austenitic stainless steel. Multi-pass butt welds were produced using the filler material SAS4-IG (Böhler Welding Austria GmbH) by manual welding with the TIG process. Spectral analysis allowed for obtaining the mass share (in parentheses) of the following elements in iron (Fe): $\mathrm{Cr}(19 \%), \mathrm{Ni}(11.5 \%), \mathrm{Mo}(2.8 \%), \mathrm{Mn}(1.4 \%), \mathrm{Si}(0.8 \%), \mathrm{C}(0.035 \%)$. A smaller section of the whole sample measuring $293 \times 40 \times 13 \mathrm{~mm}^{3}$ and containing the weld in the middle, was used for further examination. The weld region at the cross-section side was polished improve the optical reflection for the laser vibrometer measurements.

The experimental setup, including the Laser Doppler Vibrometer (Polytec OFV-5000) used for all wave propagation measurements, is depicted in Fig. 1. The ultrasonic waves were emitted by the Krautkaemer probe UWB2, a $2 \mathrm{MHz}$ variable angle beam probe set to $45^{\circ}$ long wave generation in steel. The probe was positioned such that the whole weld area was covered by the longitudinal wave. The LDV head (Polytec OFV-353) detected the out-of-plane displacement. The weld cross-section was scanned mechanically over the $x-y$ plane by moving the vibrometer head using a scanning table. The step width was $50 \mu \mathrm{m}$, so the scan size of $400 \times 240$ points yielded a scan area of $20 \times 12 \mathrm{~mm}^{2}$. The scanning table offered precision better than $10 \mu \mathrm{m}$. During the measurement, the laser beam was kept strictly perpendicular to the polished sample surface to achieve maximum laser beam reflection. For a high signal-to-noise-ratio, the improved averaging [16] was applied, enabling 10,000 measurements per scan point to be collected. The averaged time signals were stored for all $400 \times 240$ scan points, giving a set of scalar values over a $3 \mathrm{D}(2 \mathrm{D}$-space $\times 1 \mathrm{D}$-time) volume. The data were evaluated according to the GIUM workflow, as described in section Section 2.2 .

After the measurements, a $50 \mathrm{~mm}$ long and $3 \mathrm{~mm}$ thick part, containing the weld, was cut off one specimen by spark erosion (Fig. 1). It was electrochemically polished to remove the most upper surface layer which had been mechanically deformed by the polishing process and scanned using the EBSD method (EDAx 
a)

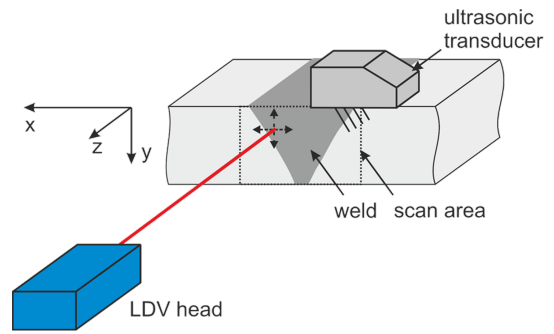

b)

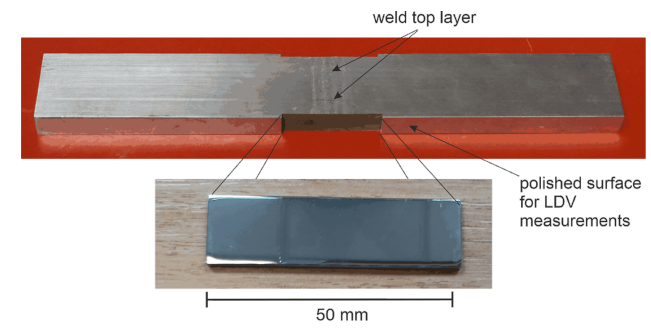

Figure 1: Experimental GIUM: a) schematic diagram of the LDV measurement (from [17] with permission of AIP); b) sample machined out of a welded plate for the LDV measurements and the piece for EBSD measurement cut out by spark erosion.

DigiView system) to determine crystallographic orientations. The scan output 88 scan areas of a size of approximately $2.8 \times 2.8 \mathrm{~mm}^{2}$ each and the pixel size was $10 \mu \mathrm{m}$.

\subsection{The workflow for generating GIUM images}

Previous publications [18 20] on GIUM mention two procedures for getting images. A simple one works only for straight wavefronts and does essentially a re-slicing of the data volume. We concentrate here on the second approach which is coming itself in two variants the 'amplitude image' and the 'time image'. This approach was described in the previous publications only very general, so we explain them here a bit more detailed.

Each pixel of a GIUM image corresponds to a measurement point (or a node in FE simulations). First, the algorithm searches for the first zero-crossing of the time trace recorded at each pixel. The time of the first zero-crossing is written to a matrix $\mathbf{M}_{1}$ (which has the dimensions of the image). A two-dimensional Gaussian filter is then applied to $\mathbf{M}_{1}$, yielding a new matrix $\mathbf{M}_{2}$. In the analysis below, we used a standard deviation of $\sigma=2$ pixels and truncated the filter after 3.5 $\sigma$, resulting in approximately 15-by-15 pixels filtering window. The high-pass filtered variant $\mathbf{M}_{3}$ (equal to $\mathbf{M}_{1}-\mathbf{M}_{2}$ ) is saved as a 'time image'. The 'amplitude image' is formed by recording values of the displacement at time instants in $\mathbf{M}_{2}$ (a low-pass filtered version of $\mathbf{M}_{1}$ ). To enhance the resolution, we used local linear interpolation along the time axis.

For comparison, we also used another variant of this algorithm, which is based on the first peak, rather than the first zero-crossing of the time trace. The 'peak' variant required further spatial Gaussian filtering of the amplitude image to produce an convenient image and emphasise the variations of peak amplitudes around their mean across the whole domain.

\subsection{Images obtained from experiments}

The GIUM processing workflow was applied previously to experimental data, as reported, e.g. in [20]. Here, we reproduce these images for reference. They were generated using the 'zero-crossing' procedure based on time traces measured with the setup described in Section 2.1. Figure2 2 presents two images obtained with different orientation of the primary longitudinal wave, that is $45^{\circ}$ and $-45^{\circ}$ from the sample upper surface normal. Both images show the grain structure of the weld clearly, while specific differences in the detailed 

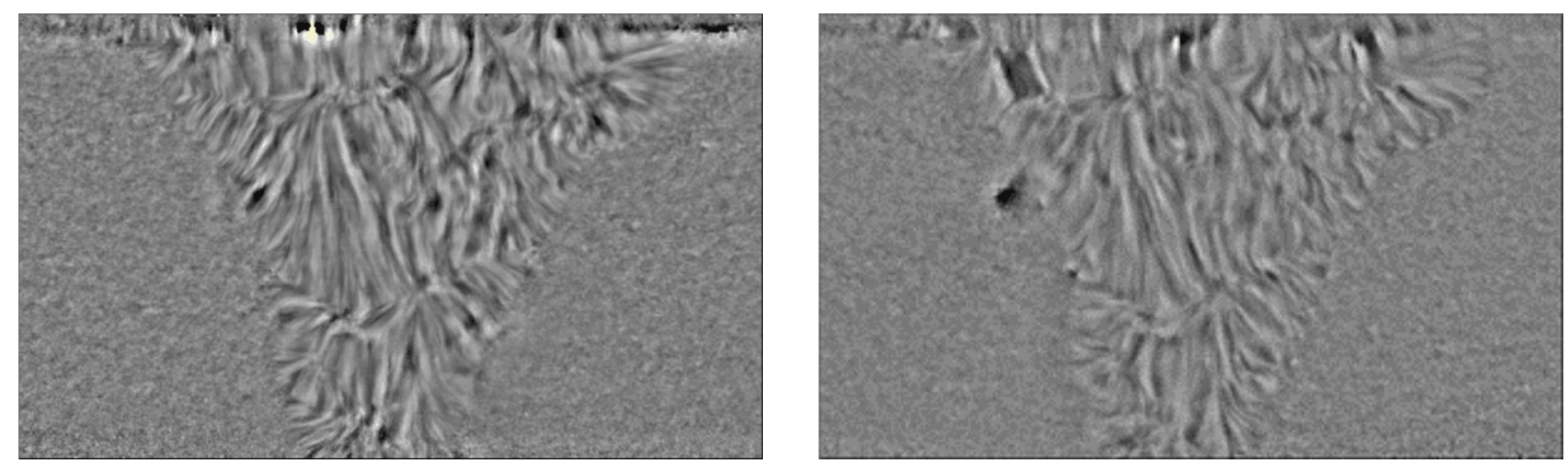

Figure 2: Experimental GUIM zero crossing time images obtained from a two $2 \mathrm{MHz}$ longitudinal wave incident at $45^{\circ}$ from the upper left side (left image) and the upper right side (right image). The position of the exciting probe was above the image such that the centre of its active area was about $5 \mathrm{~mm}$ from the middle line of the image on the respective side. The size of both images is $20 \times 12 \mathrm{~mm}^{2}$.

representation (the distribution of the grey values) exist. In this paper, we use an excitation configuration analogous to that of the right-hand image in Fig. 2, so this result should be used for comparison with images based on numerical simulation.

\subsection{EBSD data synthesis}

The microstructure used in the finite element model of the current study was synthesised directly from the EBSD measurement, offering a reliable representation of the specimen. A brief description of the reconstruction process is given below. The sample was relatively large, so the EBSD scan required dividing the area of interest into nearly 90 small tiles. The position and the overlap between tiles were first determined manually, based on the inverse pole figure colour maps generated for each dataset. While there exist tools allowing for some degree of automatisation of the process, they do not perform well if the overlap between tiles is not uniform.

The stitching was performed on raw EBSD datasets, based on the coordinates of tiles obtained from arranging the tiles manually. The microstructure was reconstructed from the stitched image using DREAM.3D [32], a freely available package for microstructure synthesis. The chosen reconstruction parameters promoted larger grains and disregarded smaller grains (in particular those in the parent material), to simplify the model and allow the features of interest to be dominant. We chose allowable misorientation of 8 degrees while segmenting the grains and required the grain to be composed of at least 60 voxels (each voxel was a $10 \times 10 \mu \mathrm{m}$ square). Such a set of parameters would typically make smaller grains and other fine details vanish during the reconstruction. However, it also allowed us to reach a balance between artefacts related to the quality of the data and the requirements of ultrasound simulations. Fig. 3 shows the synthesised microstructure. The areas around the weld were expected to behave as an isotropic solid in a macroscopic sense. Hence, in all further simulations, these regions are modelled as homogeneous isotropic. The area-weighted average length and width of the columnar grains (given by the best-fit ellipses, of aspect ratio smaller than 0.5) dominating 
the weld were 0.8 and $0.2 \mathrm{~mm}$, respectively. The less indicative area-weighted average equivalent diameter of these grains (assuming they are circles) was $0.4 \mathrm{~mm}$.

For each synthesised grain, DREAM.3D calculated average Euler angles. In most crystallographic analyses (and in DREAM.3D), passive Euler angles are defined as the angles bringing the sample reference frame to the crystal reference frame. FE simulations of ultrasound require an inverse definition - material properties should be given in the sample reference frame. Therefore, the elastic tensor rotation needed to align the crystal reference frame with the sample reference frame (the global FE coordinate system). This was accomplished by converting the default output Euler angles to quaternions and conjugating them.

\subsection{Material properties}

In all subsequent analyses, the grains were assumed to be characterised by an elastic tensor of a cubic structure, corresponding to stainless steel:

$$
\mathbf{C}=\left[\begin{array}{cccccc}
206.7 & 134 & 134 & & & \\
134 & 206.7 & 134 & & & \\
134 & 134 & 206.7 & & & \\
& & & 113.5 & & \\
& & & & 113.5 & \\
& & & & & 113.5
\end{array}\right] \mathrm{GPa}
$$

and the parent material was assumed to be isotropic:

$$
\mathbf{C}=\left[\begin{array}{llllll}
257.7 & 107.3 & 107.3 & & & \\
107.3 & 257.7 & 107.3 & & & \\
107.3 & 107.3 & 257.7 & & & \\
& & & 75.2 & & \\
& & & & 75.2 & \\
& & & & & 75.2
\end{array}\right] \mathrm{GPa}
$$

Both materials had the same density of $7958 \mathrm{~kg} \mathrm{~m}^{-3}$. The material properties were not measured but were assumed based on the knowledge of the materials in the sample, and they are in the range typical for face-centred cubic austenitic stainless steel crystals (see 33 for reference).

\section{Two-dimensional FE simulations}

\subsection{Description}

The 2D model was generated directly based on the reconstructed microstructure. Since the staircasing effect does not affect the ultrasound significantly in FE simulations 34, the mesh consisted of regular linear quadrilateral elements (plane strain, CPE4). For simulating ultrasound in polycrystals, two criteria 


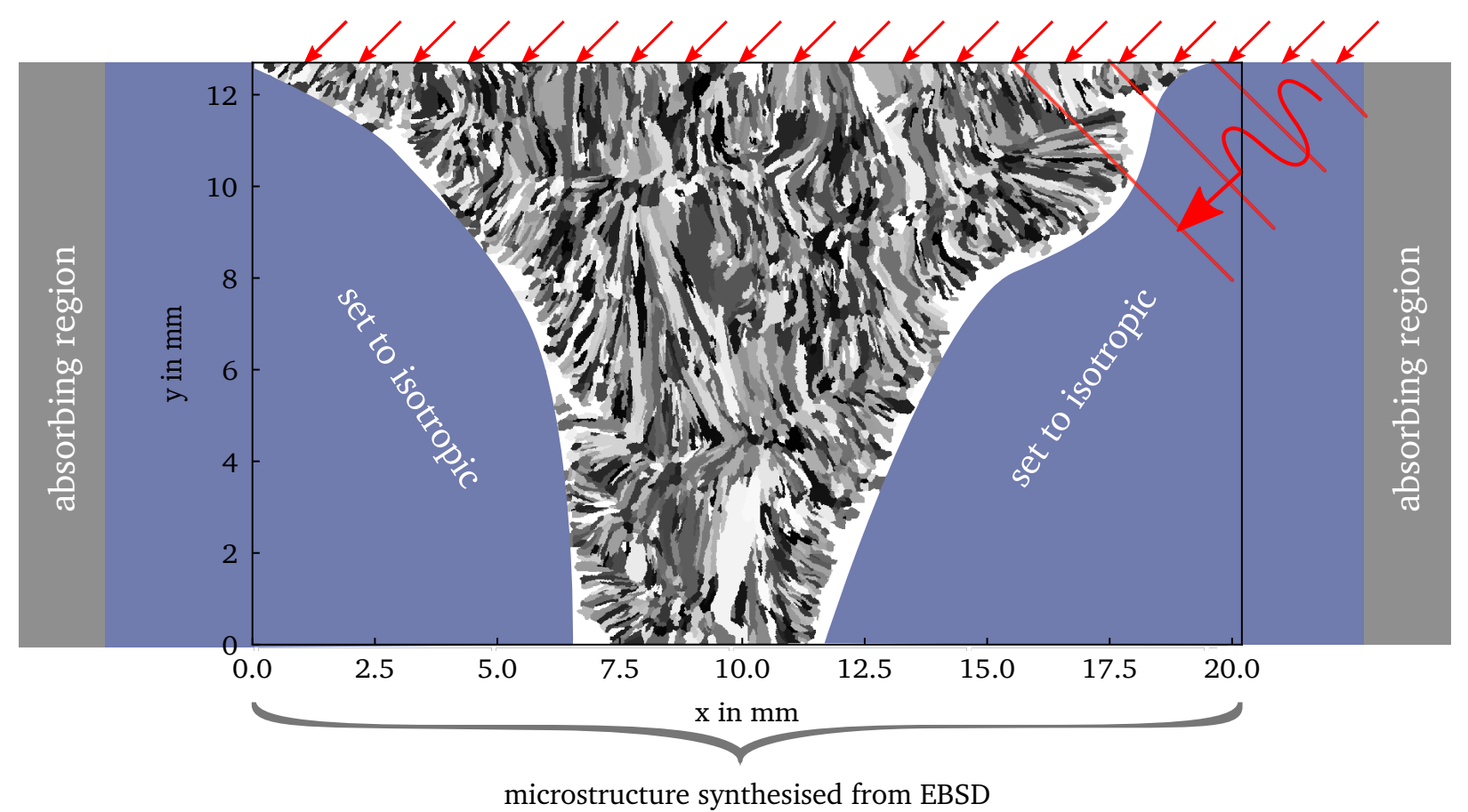

Figure 3: The schematic description of the 2D FE model, showing the microstructure synthesised from the EBSD data.

dictate the required element size; the first is its relationship to the propagating wavelength (20 elements per wavelength is a rule-of-thumb value for high-quality representation), the second - to the average grain size (at least ten elements per grain size) [22. At $2 \mathrm{MHz}$, the propagating wavelength is approximately $2.8 \mathrm{~mm}$, so the first criterion is straightforward to satisfy, given that the synthesised microstructure is output over a $0.01 \mathrm{~mm}$ grid. The second criterion is unlikely to be met here, especially for the smaller grains. However, it was primarily used in the context of precise simulations of wavespeed and attenuation in a polycrystal and is expected to be of lesser importance to GIUM-related modelling.

The first FE model is built with a $0.01 \mathrm{~mm}$ mesh (the same size as the EBSD scan resolution). In the course of this work, we also use larger element sizes to investigate the impact of the element size on the ability to model GIUM imaging.

The schematic of the FE model is shown in Fig. 3. The parent material parts to the left and the right of the weld were extended over the size of the EBSD image. This enabled the excitation to be applied over a longer distance so that a straight wavefront is achieved. In the experiment, the transducer was placed on a fluid-coupled wedge, and only the displacement normal to the surface was transmitted. We used a simplified excitation representation in the model to achieve an approximately plane wave propagating at $45^{\circ}$ to the vertical. A series of nodal forces were applied to each node between $x=0$ and $x=26 \mathrm{~mm}$, with appropriately calculated delay laws. All nodal force vectors had the same direction as the intended wave propagation direction - 45 degrees from the horizontal - to maximise the amplitude of the excited $\mathrm{P}$ 
a)

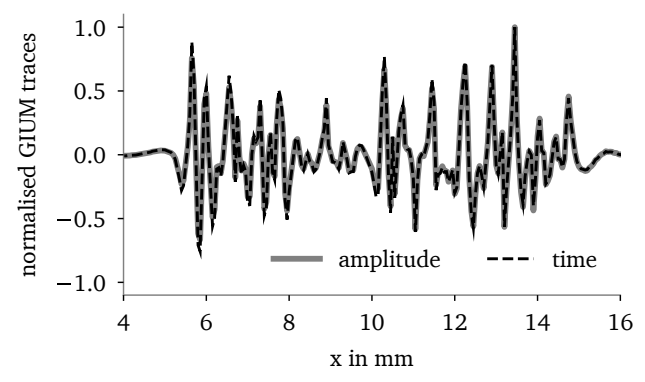

b)

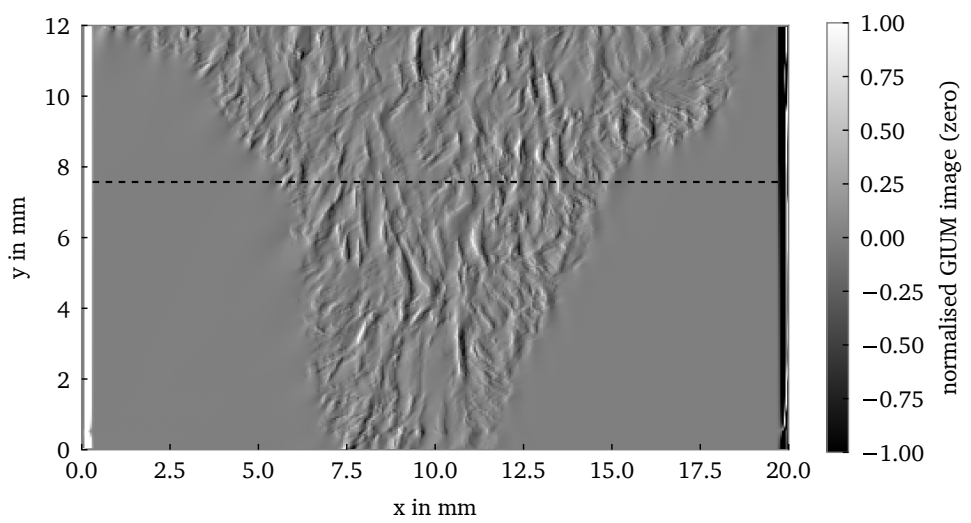

Figure 4: GIUM image based on the in-plane displacement projected along the wavefront normal: a) a slice through normalised GIUM images generated with the zero-crossing routine ; b) normalised amplitude image (slicing line marked).

wave. The excitation signal was a Ricker wavelet centred at $2 \mathrm{MHz}$. The time step was 1 ns, which was more conservative than the Courant-Friedrichs-Lewy (CFL) criterion, and the simulation ran for $6 \mu$ s. For convenience, we used absorbing regions minimising the waves incident on the left and the right boundary of the model.

The receivers were arranged into a $400 \times 240$ grid, analogous to that used in the experiment. At each point, both the $u_{x}$ and the $u_{y}$ response were recorded in a $2 \mathrm{D}$ simulation. The total number of degrees of freedom for this model was 10.75 million, and it was built for Pogo [35], a GPU-based finite element solver developed at Imperial College London [21, and solved on a GeForce GTX 1080 Ti card.

\subsection{Results}

The experimentally obtained GIUM images are based on the out-of-plane response recorded over the cross-section of the weld. In a $2 \mathrm{D}$ simulation, this component is not available, hence other field quantities need to be investigated. We considered the resultant in-plane displacement magnitude $\left(\sqrt{u_{x}^{2}+u_{y}^{2}}\right)$, the displacement projected along the wavefront normal $\left(\simeq 45^{\circ}\right)$ and the dilatation strain $\left(\mathrm{d} u_{x} / \mathrm{d} x+\mathrm{d} u_{y} / \mathrm{d} y\right)$. In addition to the presentation of obtained GIUM maps, we illustrate the differences and similarities of the different variants of the GIUM processing routines described in Section 2.2 .

\subsubsection{Displacement}

First, let us compare the time and amplitude images obtained from the 'zero-crossing' GIUM processing routine for the in-plane displacement projected along the wavefront normal. A closer inspection of the outputs allowed us to notice that if we normalise the output with respect to its maximum, the time and amplitude images will be almost identical. Figure 4 a shows slices across the same vertical position of the image for normalised time and amplitude images. Clearly the differences between the two lines are negligible, indicating that for the 'zero-crossing' variant, one can use either normalised time or amplitude image. 


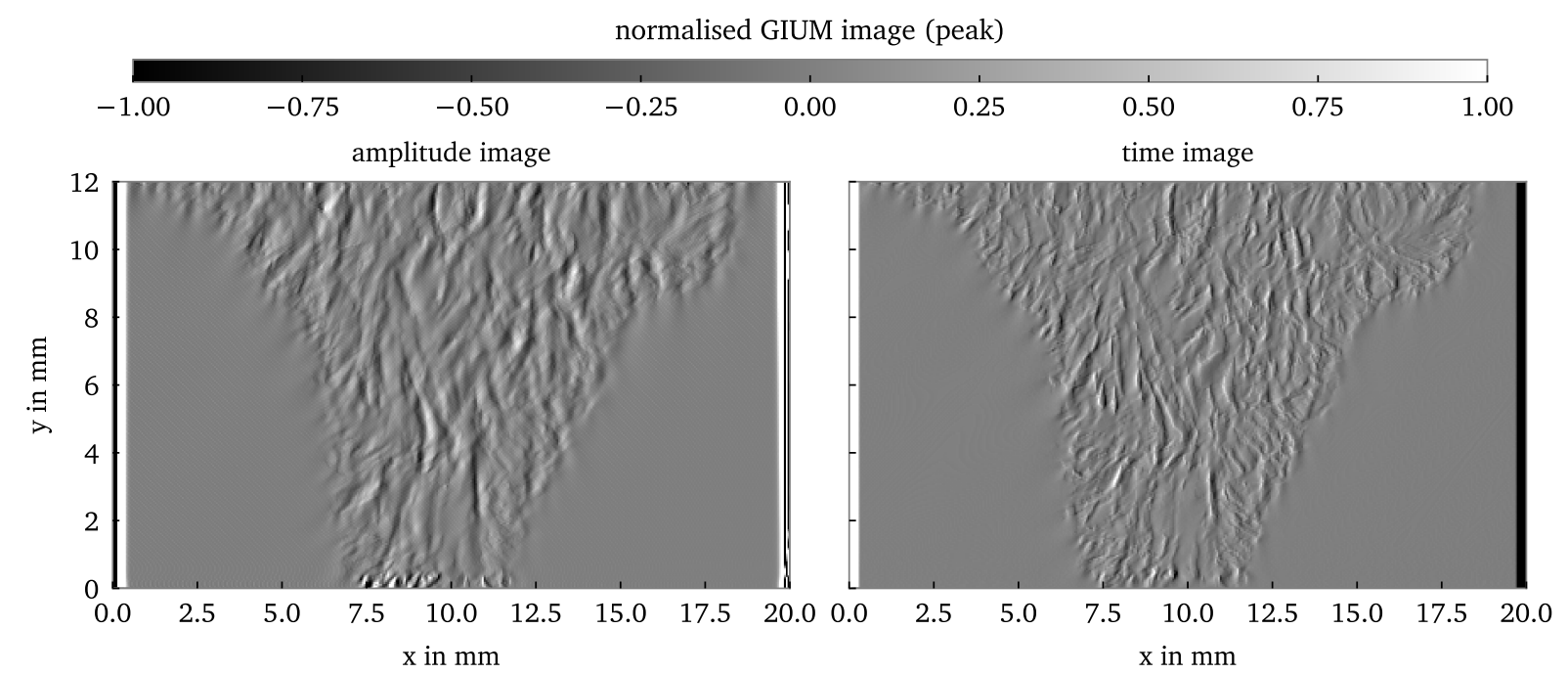

Figure 5: Normalised GIUM images based on the magnitude of the resultant displacement computed using the 'peak' routine. Both subfigures share the same $y$-axis.

The amplitude GIUM image for the projected in-plane displacement computed using the 'zero-crossing' routine is shown in Fig. 4p. While this image does not offer sharp grain boundaries, the microstructure signatures may be observed, especially the orientation of larger grains. We recall that the image obtained from the experimental data 20] was significantly clearer; however, here we are dealing with a simplified model with only surface microstructure data and infinite in the depth direction, but still, microstructure images can be generated.

The 'zero-crossing' routine is not suitable for the magnitude of the resultant displacement, as this quantity is always positive. For this reason we focus on the first peak, modifying the GIUM routine slightly. This time the normalised amplitude and time images would not be the same - the amplitude image has a further high-pass filter, so that amplitude variations around the 'average wavefront peak' can be revealed. It is worth noting that the time images for all the displacement-based calculations in this section would overlap - the time of the first peak changes in the manner as the time of the first zero-crossing. Likewise, the amplitude images computed using the 'peak' routine will also overlap. Hence, for brevity, we only plot the time and amplitude images for the resultant displacement magnitude in Fig. 5 . Although certain similarities exist, the time image is sharper, with better-pronounced grain boundaries and more detail. This difference indicates that the time of the peak is more sensitive to the local property changes than its amplitude, unlike the relationship observed in the neighbourhood of the zero-crossing.

\subsubsection{Strain}

The experimentally obtained GIUM image is based on the out-of-plane displacement, which is not available for a 2D simulation with plane strain elements. However, one could expect that the out-of-plane 


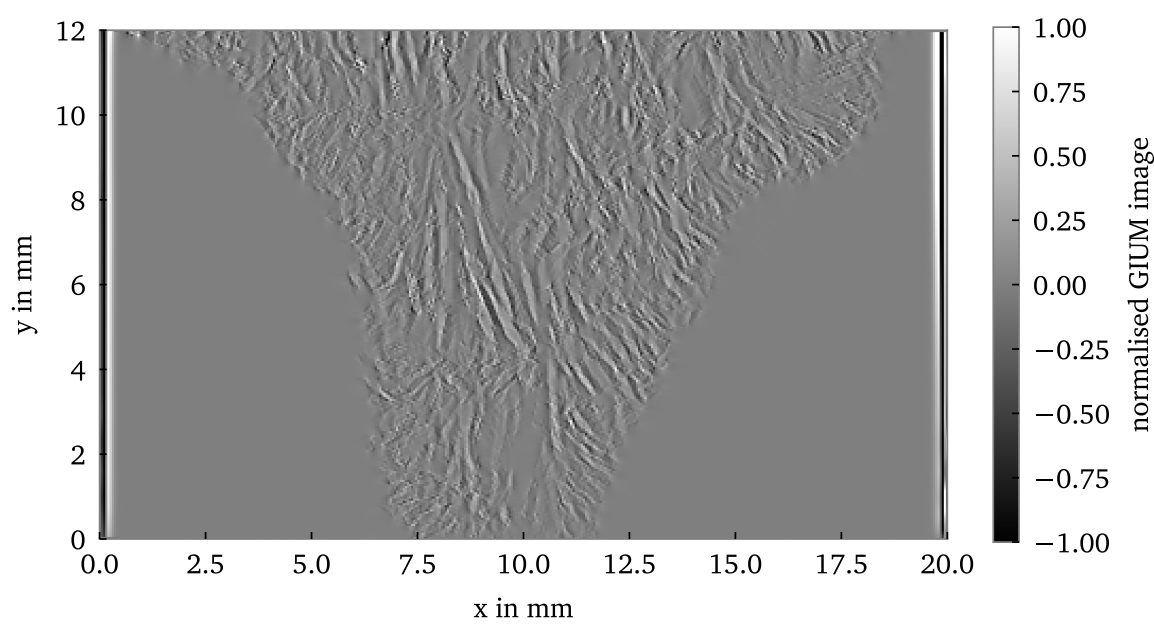

Figure 6: Normalised GIUM amplitude image based on the dilatation strain, computed using the 'peak' routine.

displacement is in part related to the lateral contraction and the resultant stress, following the in-plane loading. Let us recall Hooke's law relating stress $\sigma_{i j}$ to strain $\epsilon_{i j}$ :

$$
\sigma_{i j}=\lambda \delta_{i j} \Delta+2 \mu \epsilon_{i j}
$$

with $\lambda$ and $\mu$ being the Lame parameters, $\delta_{i j}$ the Kronecker delta function and $\Delta=\epsilon_{11}+\epsilon_{22}+\epsilon_{33}$ - dilatation. In a plane strain model, only a portion of the components of the stress tensor are available and the lateral contraction only manifests itself in the dilatation component. The dilatation represents fractional volume change, which may be intuitively expected to form a link between the 3D observations and 2D simulations.

Hence, we now focus our attention on the dilatation strain, which under the plane strain assumption is defined as:

$$
\Delta=\mathrm{d} u_{x} / \mathrm{d} x+\mathrm{d} u_{y} / \mathrm{d} y
$$

In this paper, spatial derivatives of the displacements are calculated numerically from the displacements, using second-order accurate central differences (as implemented in Numpy [36]). Spatial differentiation will act as a simple edge detector, so one can expect that the grain boundaries would be better pronounced. Further, the structure of strain time traces is slightly different - their zero-crossing would correspond to the peak of the displacement, with the strain peak leading the displacement peak along the time axis. Hence, for GIUM images based on strain, we used the 'peak' routine and focused on the amplitude image, which both produce better results than their counterparts.

The dilatation-based GIUM image in Fig. 6 shows more detail than the displacement images. One plausible reason links to the edge-detecting function of the spatial derivative that was mentioned above. The improvement over the displacement-images still falls short of the quality of the experimentally acquired figure. 
Nevertheless, we demonstrated that the numerical simulations based on the synthesised microstructure can capture complex wave behaviour in the weld and yield results sufficient to derive an image of the microstructure using the GIUM procedure.

\subsubsection{The effect of the mesh size}

Having identified the most plausible quantity for GIUM imaging from in-plane displacements, let us now focus the attention on the effect of the mesh size in the light of the established criteria for polycrystal models.

While the size of the $2 \mathrm{D}$ model with $0.01 \mathrm{~mm}$ mesh does not pose any technical challenge for Pogo [21] and it can easily run on a low demand system (e.g. based on a GeForce GTX 1080 Ti card), a threedimensional model can rapidly grow to above 1 billion degrees of freedom. The criterion for designing mesh for simulating ultrasound in polycrystals (ten elements per grain) may be too restrictive for our problem. Hence, in this section, we reduce the mesh density and assess its impact on the GIUM images.

The comparison consists of the models with different mesh sizes: 0.01, 0.02, and $0.05 \mathrm{~mm}$ (corresponding to approximately 280, 140, 56 elements per wavelength). Amplitude images synthesised from numerically simulated responses are shown in Fig. 7 .
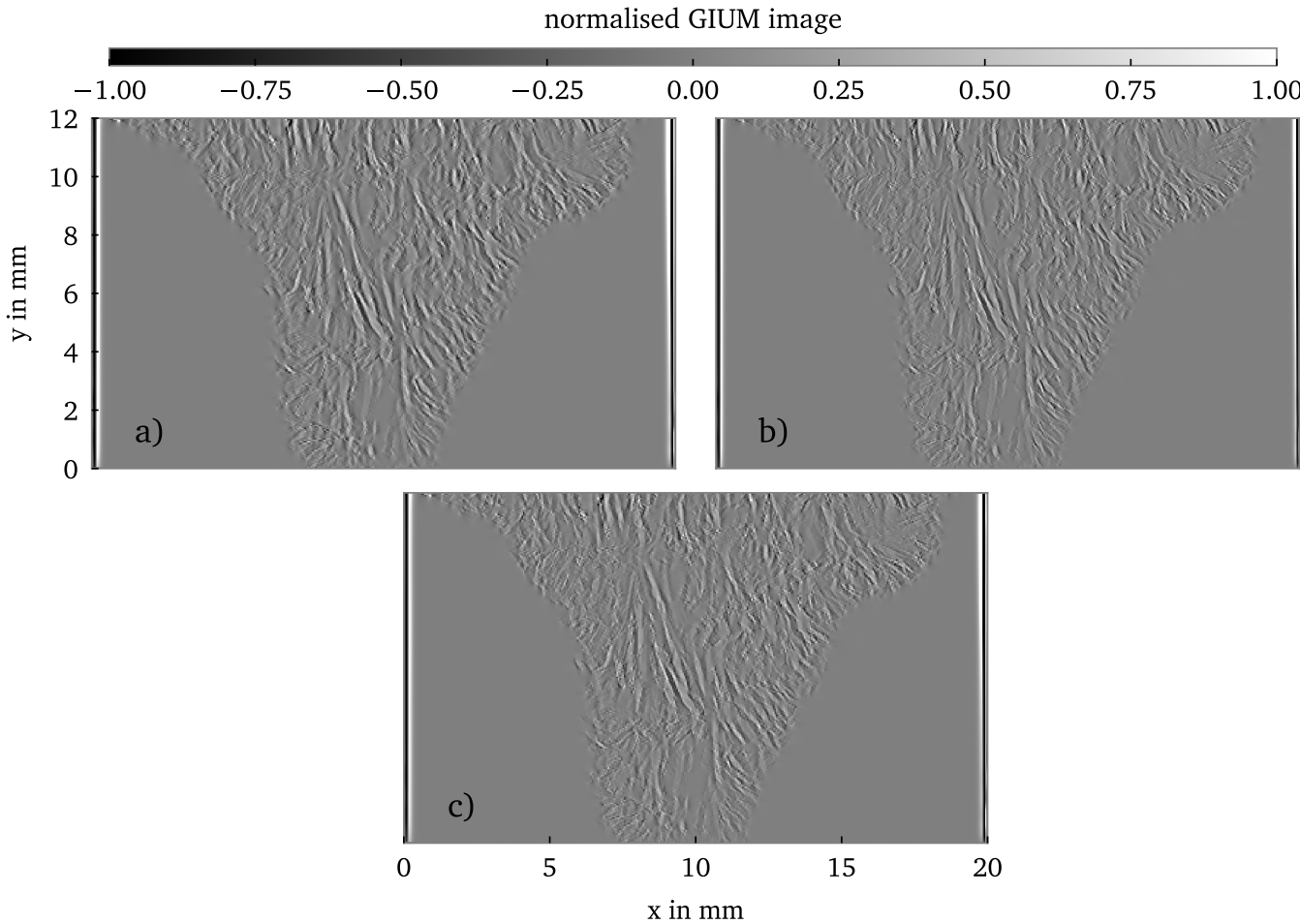

Figure 7: Normalised GIUM amplitude images (peak) based on dilatation strain, generated from simulations based on meshes with different element sizes: a) $0.01 \mathrm{~mm}$, b) $0.02 \mathrm{~mm}$, c) $0.05 \mathrm{~mm}$.

The results indicate that although the 'ten elements per grain' criterion is not always fulfilled for the 
$0.05 \mathrm{~mm}$ mesh, the differences between images are very fine and are unlikely to be of practical significance.

Thus, all three-dimensional models will use a $0.05 \mathrm{~mm}$ mesh.

\subsubsection{The effect of the excitation frequency}

In most imaging methods, higher excitation frequency provides better resolution, which for coarse-grained materials comes at the cost of an elevated structural noise level. Aware of this limitation, we simulated the ultrasound for excitation centred around $5 \mathrm{MHz}$ and $10 \mathrm{MHz} \mathrm{MHz}$, to assess how susceptible is the GIUM image generation procedure to scattering noise. Referring to Section 2.2, we recall that GIUM images do not rely on measuring the time of flight between sensors and features, and hence it is unlikely that the resolution improves with increased frequency.

The comparison between the three excitation frequencies considered in this document so far is shown in Fig. 8.

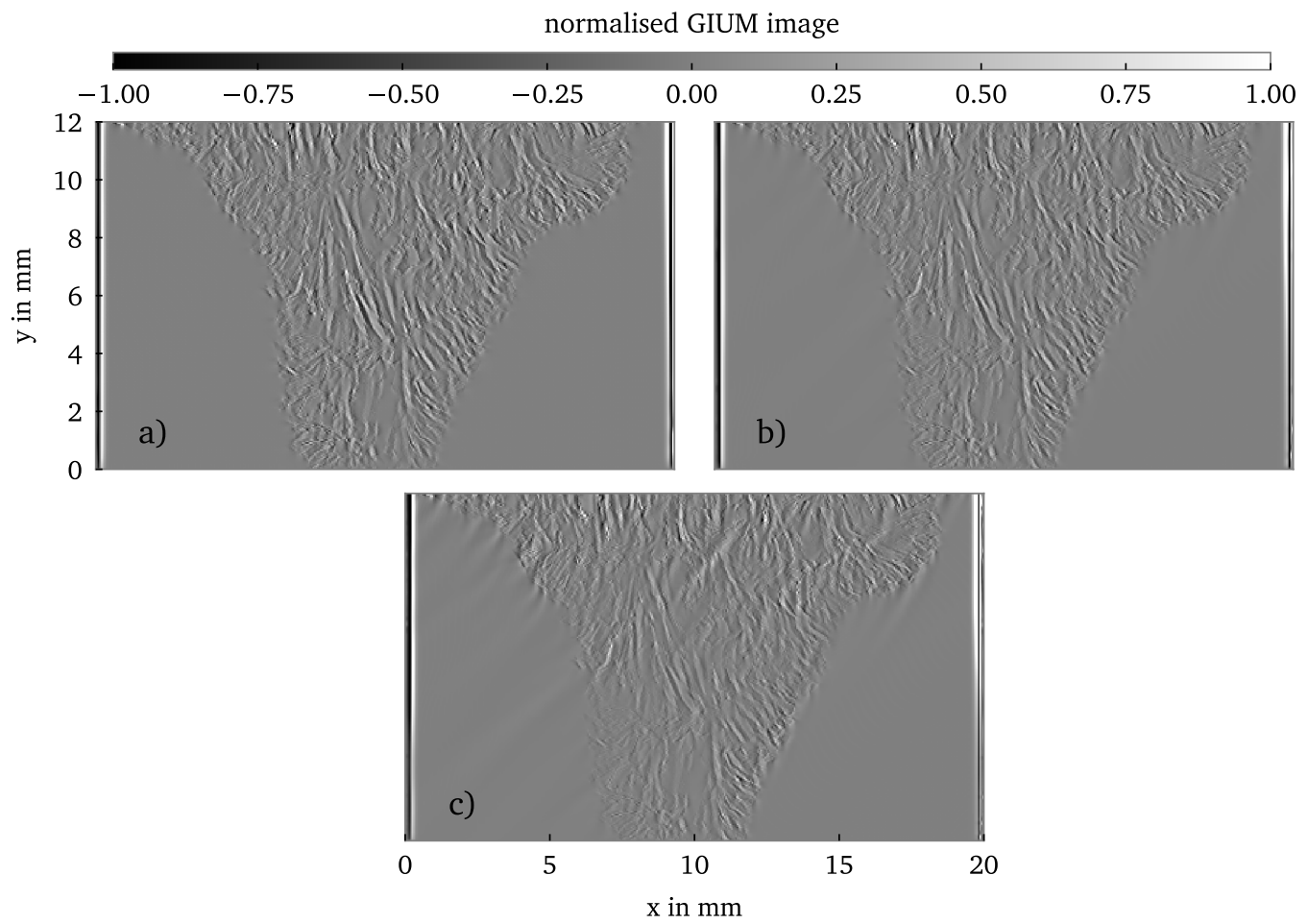

Figure 8: Normalised GIUM amplitude images (peak) based on dilatation strain, for different excitation frequencies: a) $2 \mathrm{MHz}$, b) $5 \mathrm{MHz}$, c) $10 \mathrm{MHz}$.

Several observations can be drawn. First, the level of detail does not increase with frequency, as previously indicated. Moreover, the images show a non-uniform intensity (the wave attenuates more significantly when propagating across the weld at $10 \mathrm{MHz}$ ) and the 'shadows', reasons for which have not been identified yet. A tentative explanation is that they are a consequence of the wavefront being more abruptly deviated by the microstructure, resulting in significant local variations of the arrival time of the wave. Nevertheless, 
this simple comparison indicates that there is a trade-off between the ability to track the zero-crossing or the envelope peak accurately (which is easier at higher frequencies) and the detrimental effects of highfrequency excitation (attenuation, non-uniform wavefront). The comparison presented here indicates that GIUM imaging is better suited to lower frequencies.

\subsubsection{Resultant out-of-plane stress}

The dilatation strain was proposed in Section 3.2 .2 as a link between the 2D simulations and real, 3D experimental observations. In this section, we follow that intuition further, assuming that the out-of-plane displacement at the free surface in a 3D model of elongated 2D grains is related to the out-of-plane normal stress $\sigma_{z z}$ in the 2D model. The latter should be equivalent to a 3D model for $x_{z}<0$ with an identical grain structure (extruded grains) and surface conditions: $u_{z}=0, \sigma_{x z}=\sigma_{y z}$. From Hooke's law, $\sigma_{z z}$ in the 2D model can be calculated as:

$$
\sigma_{z z}=c_{31} \epsilon_{x x}+c_{32} \epsilon_{y y}+c_{33} \epsilon_{z z}+c_{34} \epsilon_{y z}+c_{35} \epsilon_{x z}+c_{36} \epsilon_{x y}
$$

Under the plane strain assumption, all derivatives over $z$ vanish. In this paper, we neglect the contribution of the shear strain $\epsilon_{x y}$. The out-of-plane stress is then calculated as:

$$
\sigma_{z z} \approx c_{31} \epsilon_{x x}+c_{32} \epsilon_{y y}
$$

Based on the above relationship, we calculated $\sigma_{z z}$ from 2D numerical simulations. The stress calculation followed the standard procedure for displacement-based FE. The stress was first calculated at the integration points, from strains following the assumed shape functions (linear elements) and their derivatives and element stiffness matrices. The 'element stress' was then extrapolated from the integration points to the nodes. Finally, stress coming from all elements which share a node were averaged.

The GIUM image based on the resultant $\sigma_{z z}$ stress is shown in Fig. 9- this is the 'peak' variant which allowed for obtaining the best quality image.

This image is a perfect representation of the microstructure used to develop the FE model. The resultant stress $\sigma_{z z}$ is in a reasonable approximation solely governed by the Poisson's effect, and yet, it unexpectedly yields a high-quality image. The lateral contraction may play a role in exposing the microstructure signatures in the experiment, as well; however, given the amount of simplifications required for the $2 \mathrm{D}$ plane strain model, the evidence presented above is not sufficient. In the following sections we attempt to explain the physical mechanism further.

\section{Three-dimensional FE simulations}

Three-dimensional simulations are the closest replica of the experimental setup. However, a single EBSD scan offers no information regarding the grain structure underneath the surface, and the calculation is bound 


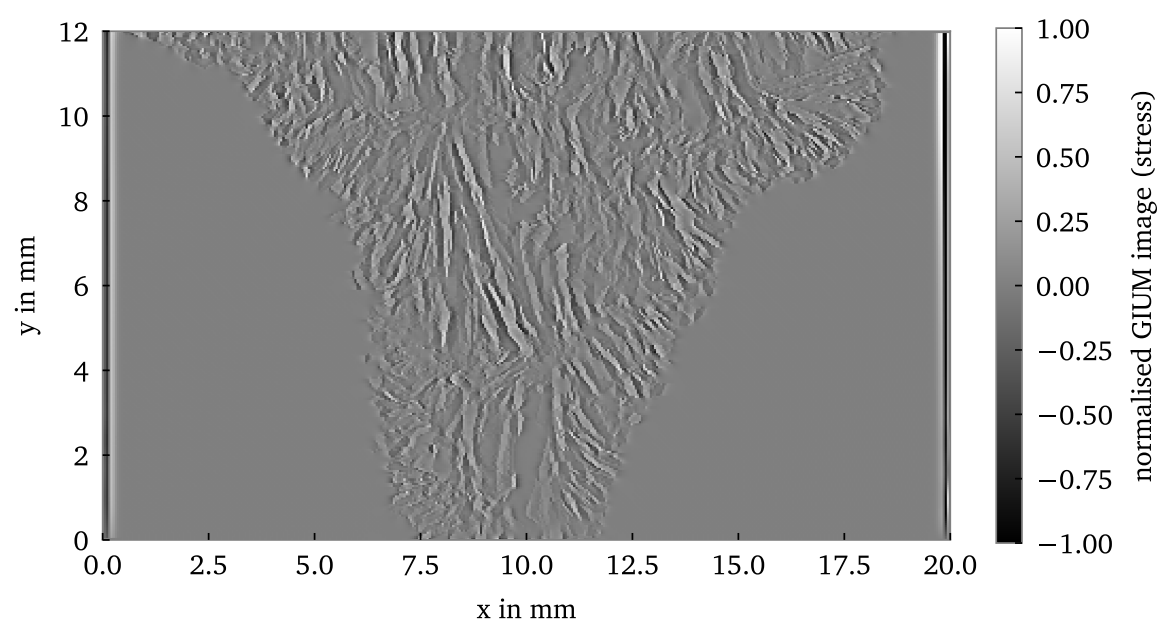

Figure 9: Normalised GIUM amplitude image (peak) generated from $\sigma_{z z}$ stress.

by an assumption that the GIUM image is affected only by grains adjacent to the surface. This assumption will be further assessed in Sect. 6. In this section, we use a simplified representation of grains in 3D.

\subsection{Description}

The 3D model is constructed by extruding the 2D synthesised microstructure in the depth direction $(z)$. The model is uniform in the $z$ direction, and crystallographic orientations are the same as those used in the 2D counterpart. Following from the mesh size study in Section 3.2 .3 , the mesh density could be reduced as compared to the EBSD grid. The element size is $0.05 \mathrm{~mm}$, and the overall thickness of the model is $6 \mathrm{~mm}$. The elements are now 8-node regular hexahedra (C3D8 family). The 3D model has just over 77 million degrees of freedom and was solved using Pogo [21, 35] on four Nvidia GeForce RTX 2080 Ti cards. The solution time for 6000 steps was around 6 minutes.

Like before, we added absorbing regions at the left and right extremities of the model to minimise reflections. All elements outside the weld are set to be isotropic, for simplicity. The structure is excited by a plane-like wave originating from the top-right corner. The excitation is a set of point forces acting at $45^{\circ}$ to the vertical and subject to appropriately calculated delay laws so that the resultant wave crest travels at $45^{\circ}$ to the horizontal. At each forcing node position over the $x-y$ plane, we applied the same delay for all nodes along the depth direction. The loading signal is a Ricker wavelet centred around $2 \mathrm{MHz}$. Finally, the receivers were arranged over the $z=0$ plane in a regular $400 \times 240$ grid, recording both the in-plane $(x-y)$ and the out-of-plane $(z)$ displacement.

\subsection{Results}

Based on the out-of-plane displacement predicted by FE, we generated GIUM images using the workflow outlined in Section 2.2, replicating the scenario from the experiment [18]. The performance of the 'peak' 


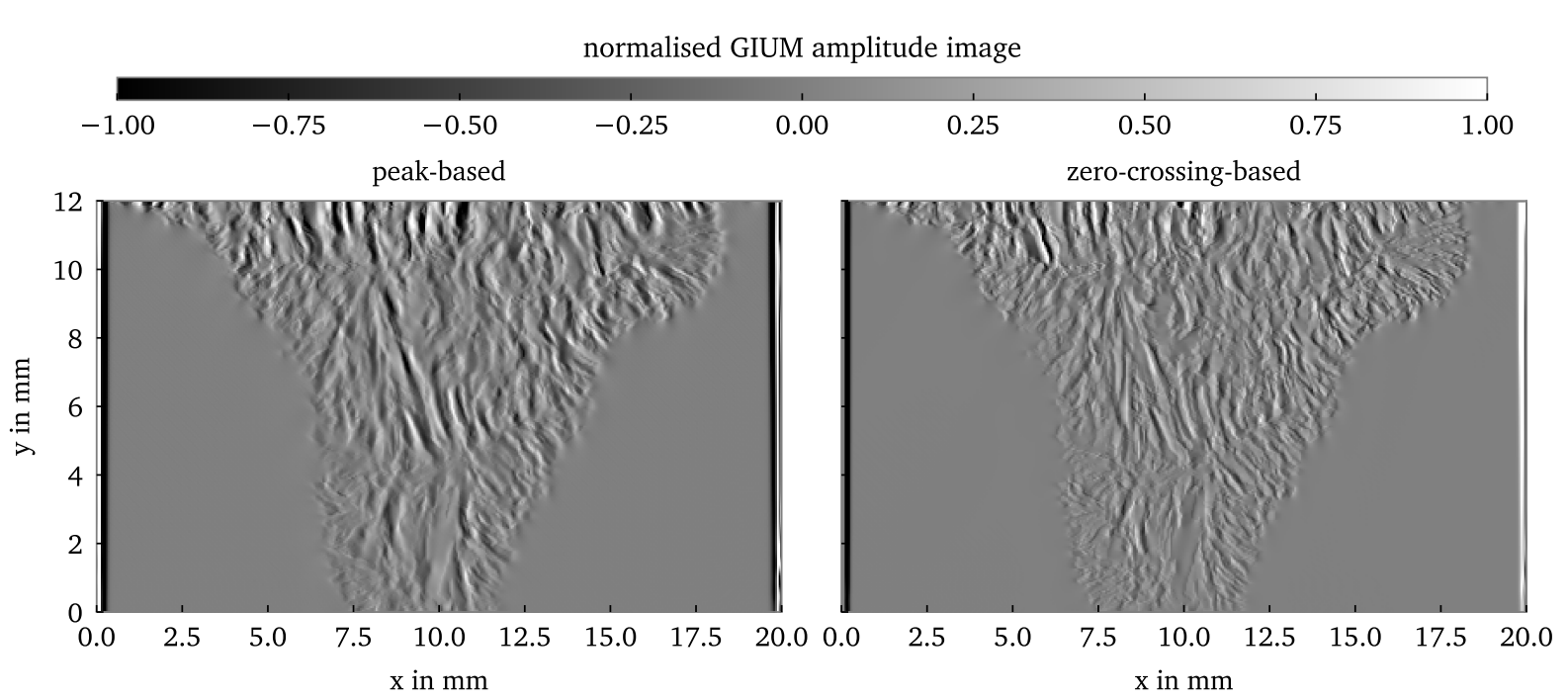

Figure 10: Normalised GIUM amplitude images obtained from 3D simulations $\left(u_{z}\right)$ using the 'peak' extraction routine (left) and the 'zero-crossing' routine (right).

and 'zero-crossing' GIUM routines was slightly different. In Fig. 10, we compare GIUM amplitude images generated based on the first peak and the first zero-crossing. The 'peak'-based image is more blurred and with some grain boundaries lost, but the signatures of grains are present and follow the expected pattern.

The image rendered using the 'zero-crossing' is richer in detail - grain patterns can clearly be distinguished, and they represent well the underlying microstructure from Fig. 3 and the experimentally acquired data presented in [20]. The numerical model is an analogy to the real scenario, and the assumptions taken when developing the simulation are shown not to compromise its physical foundation. Quite surprisingly, the difference between the 'zero-crossing' image in Fig. 10 and the dilatation-based image in Fig. 6. is rather insignificant. Further, both numerically generated images are less clear than the experimentally acquired image in [20]. A potential explanation is that the synthesised EBSD model is more complex (more smaller grains) than the actual sample. This could be attributed both to not always perfect quality of the EBSD dataset and perhaps too conservative choice for the reconstruction parameters. It is also possible that during the experiments fine local effects were averaged, allowing the larger grains to dominate the image.

Nevertheless, the results presented in this paper confirm that GIUM reveals features significantly smaller than the propagating ultrasonic wavelength. At $2 \mathrm{MHz}$, the propagating wavelength is approximately $2.8 \mathrm{~mm}$, whereas the area-weighted average length and width of the columnar grains are 0.8 and $0.2 \mathrm{~mm}$, respectively. Both the experimental and all numerical results show signatures of grains far below the wavelength, as clearly visible in the images. This is not a contradiction to the physics - GIUM does not detect scattered ultrasonic waves by an ultrasonic probe, but it is based on ultrasonic field values (here, the out of plane displacement on the free surface) recorded by a sensor with an aperture much smaller than the 
ultrasonic wavelength (laser dot, or a nodal value in simulations).

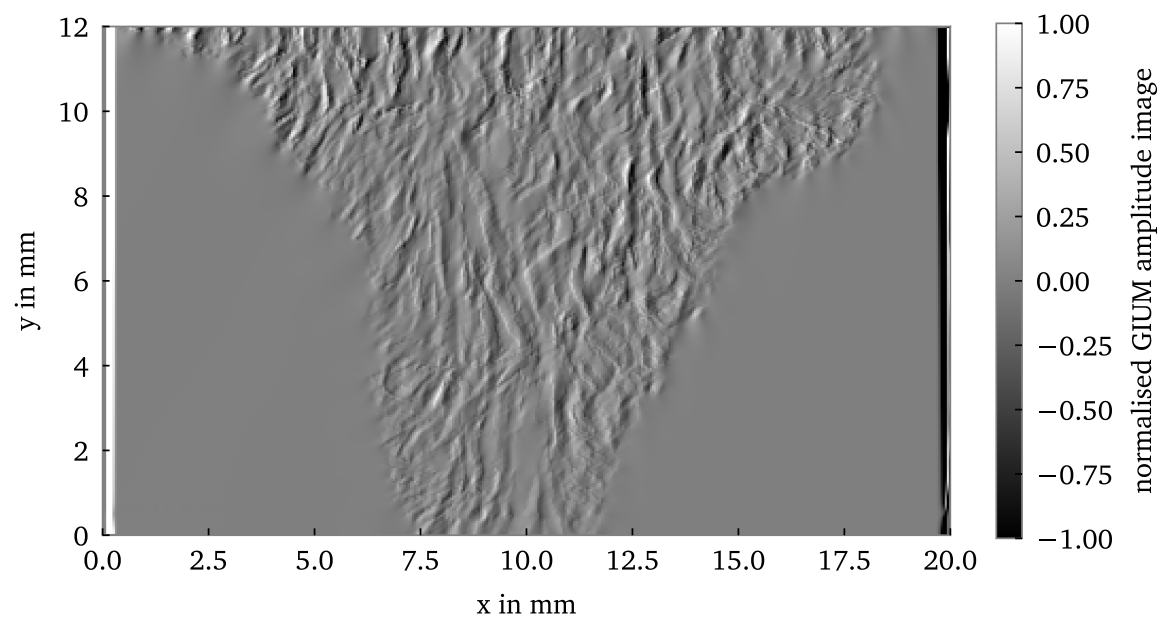

Figure 11: Normalised GIUM image obtained from 3D simulations based on the in-plane displacement.

GIUM images are formed based on the measured out-of-plane response, which is in practice much more convenient to acquire than the in-plane response. However, for completeness, we computed GIUM images from the simulated in-plane surface displacements. For this calculation, we projected both $x$ and $y$ displacement on the direction parallel to the wave vector (the wave is incident at $45^{\circ}$ ) to obtain a single time trace corresponding to the in-plane displacement. The generated GIUM image is shown in Fig. 11. While the grain structure can be recognised, upon closer inspection, the contrast and the level of detail is slightly poorer in comparison to the out-of-plane image in Fig. 10. Why the out-of-plane response provides better GIUM images? The answer is attempted in the following section.

\section{The physical mechanism behind GIUM imaging}

In the preceding sections, we demonstrated that appropriately designed grain-scale numerical simulations capture all phenomena contributing to GIUM images. Several observations indicated that both the lateral contraction and the free surface effect come to play. Since the conception of the GIUM method, we assumed that the surface-skimming longitudinal wave is responsible for the manifestations of the microstructure. This section presents a simplified numerical model to investigate this claim further.

The algorithm for generating GIUM images recalled in Section 2.2 originates from two features of the recorded signals: the spatially high-pass filtered time of the first zero-crossing (or envelope peak) and the amplitude of displacement at a time instant corresponding to the spatially low-pass filtered time of zerocrossing. The former encompasses the subtle local differences of arrival times between neighbouring locations and contributes to the 'time image'. The 'amplitude images' are the values of displacement at time instances following the 'main wavefront', regardless of the local variations of the arrival time. 


\subsection{Lateral contraction}

Sect. 3 demonstrated that the GIUM image could be generated from the resultant stress coming from $2 \mathrm{D}$ simulations. That image was solely governed by the lateral contraction and yet, corresponded very well to the EBSD examination. This result allowed us to speculate that the lateral contraction may play a role in the synthesis of the image from measured data.

The most straightforward possible configuration to validate this claim is a $2 \mathrm{D}$ model with horizontally arranged layers and the $\mathrm{P}$-wave propagating from top to bottom. One may choose to think about it as analogous to a result of cutting the 3D weld model with a plane normal to the weld cross-section and containing the incident wave vector. By looking at the response at the free surface and in 'the bulk', we capture the effects of interest. The schematic description of the model is shown in Fig. 12 .
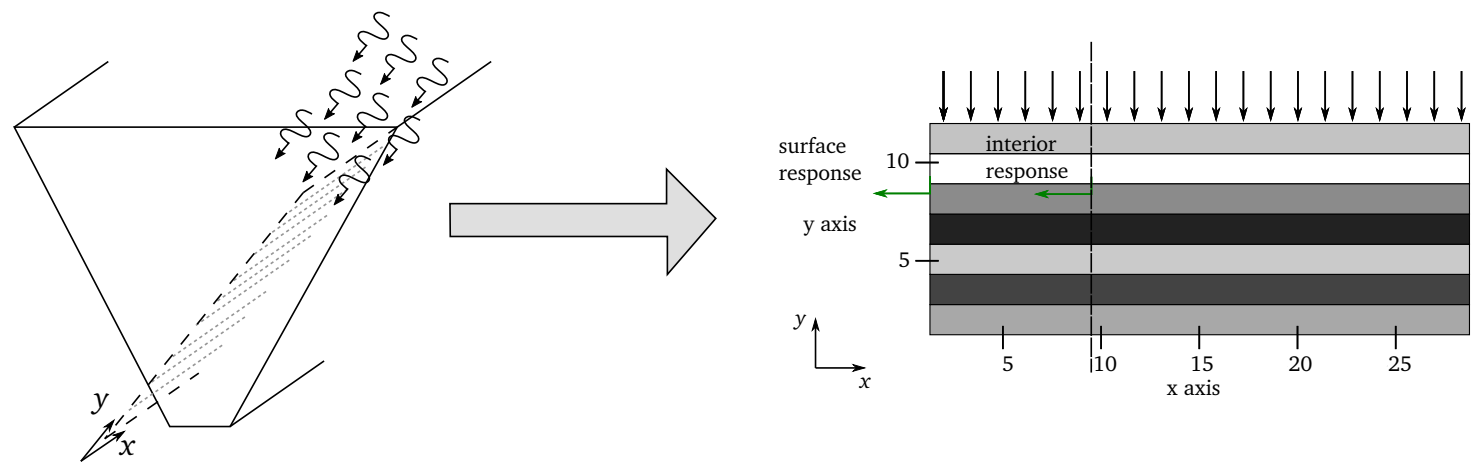

Figure 12: The 2D FE model for investigating GIUM principle; the image does not represent true layer thicknesses for clarity.

Each of the layers was characterised by a cubic elastic tensor rotated according to Euler angles, taken at random from the EBSD measurement of the weld considered in this paper. Consequently, the rotated stiffness matrices did not necessarily possess any symmetries. The frequency of excitation was $2 \mathrm{MHz}$, and the layer thickness was $0.2 \mathrm{~mm}$ (the wavelength/layer thickness ratio was approximately 14). The vertical load was applied over the whole top surface, generating a P-wave with a linear wavefront. The response perpendicular to the propagation direction (along the $x$ direction) was recorded both at the left edge of the domain (at $x=0 \mathrm{~mm}$ ) and inside (at $x=8 \mathrm{~mm}$ ).

The GIUM image extraction routine was executed over both the surface and 'interior' responses, and the result is shown in Fig. 13 a. Since we are using a single line of receivers, we refer to the result as 'GIUM trace' hereafter, as a 1D analogy to the image. Figure 13 a only shows a portion of the domain for clarity. The trace corresponding to the surface response peaks only at boundaries between the layers, which in a $2 \mathrm{D}$ context will translate to well-marked layer boundaries. The 'interior' response creates artefacts in addition to correct signatures. Moreover, these additional peaks are often dominant, allowing us to speculate that in a 2D scenario, they will significantly distort the image.

A closer inspection of the time traces revealed that away from the surface, scattering at the boundaries 
a)

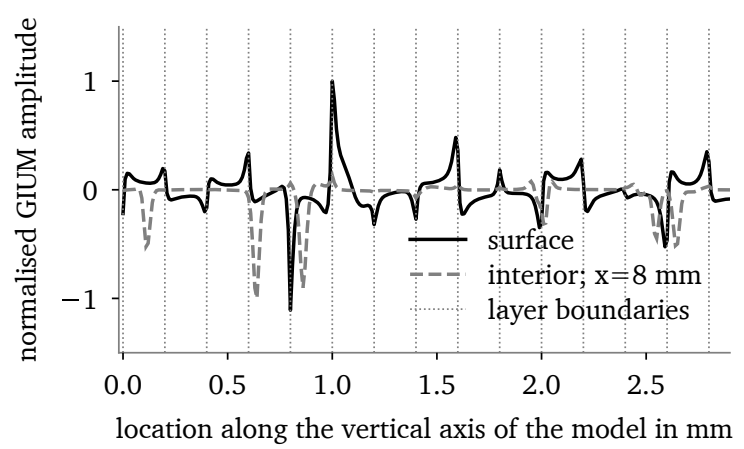

b)

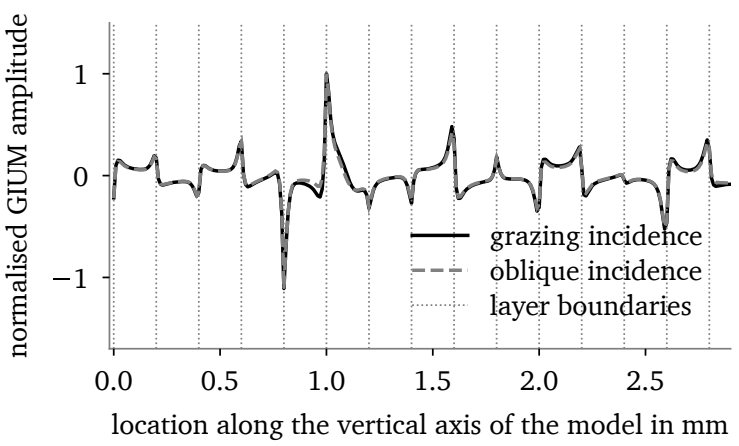

Figure 13: GIUM amplitude traces for the simplified layered model: a) comparison between the surface and the 'interior' response; b) comparison between grazing and oblique incidence; dashed vertical lines indicate layer boundaries.

\subsection{Grazing incidence}

We now turn our attention to the effect of grazing incidence and the surface-skimming wave phenomenon. A longitudinal wave travelling along a free boundary is not capable of satisfying the traction-free condition on its own. The associated surface traction can only be eliminated if a shear wave, which invokes both the normal and the transverse shear stress, exists. Hence, to balance the surface traction, the longitudinal wavefront bends slightly in the vicinity of the boundary and allows the shear wave shedding. Whether the near-surface interaction can be classified as classical diffraction has been a topic of numerous discussions in the past, e.g. in 37. Such an assumption seems justified in the context of this work. Hence, we hereafter ignore the second-order effects associated with the wavefront curvature and treat the near-boundary behaviour as a classical reflection problem.

To verify this, we computed the GIUM trace using oblique, rather than grazing incidence $\left(10^{\circ}\right)$ from the vertical). The time and amplitude GIUM traces are shown in Fig. 13b. Surprisingly, the lines are almost identical, regardless of the angle of incidence. Figure $13 \mathrm{~b}$ indicates that while the surface effect is essential, the grazing incidence is not a necessary condition. Its main advantage is practical convenience when dealing with real samples. It is worth noting that the angle of incidence can only be precisely defined at the excitation surface. While propagating across the domain, this angle will vary slightly, according to wave refraction at the interfaces between layers. We also confirm that, as suggested in Section 3 , the normalised time and amplitude traces were nearly identical, which is why only the amplitude trace is plotted. 


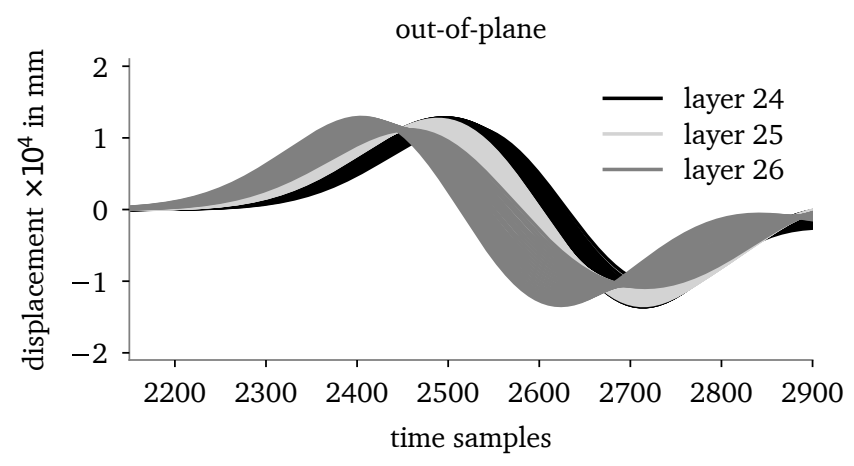

c)

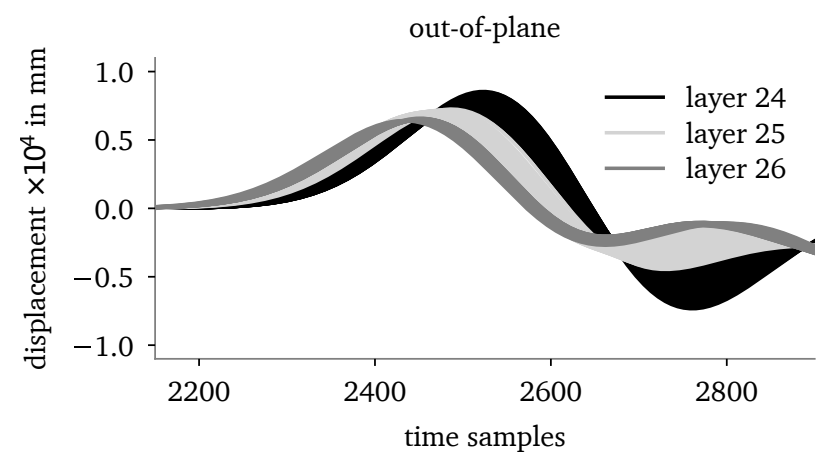

) a) b)

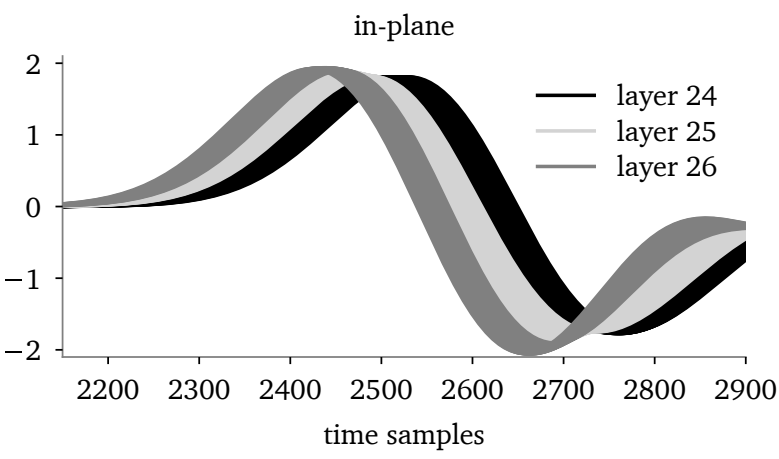

d)

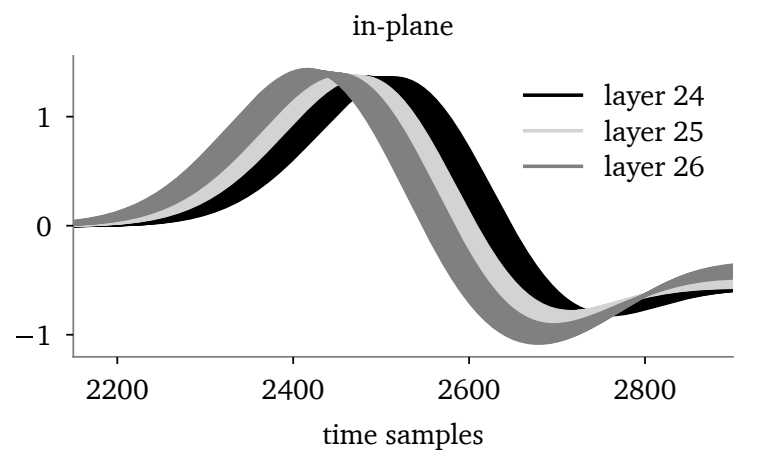

We observed in both the $2 \mathrm{D}$ and $3 \mathrm{D}$ simulated results that the out-of-plane displacement allows for generating GIUM images with better contrast and a higher level of detail. In the following paragraphs, we look closer at the features of the time traces to explain this phenomenon.
Figure 14: In-plane and out-of-plane displacement predicted at the free surface across three neighbouring layers of the: a) and b) locally anisotropic model; c) and d) locally isotropic model (the colours indicate layer indices).

Figs $14 \mathrm{a}-\mathrm{b}$ show time traces recorded at the free surface along a line spanning across three selected neighbouring layers of the equivalent layered model introduced earlier in this section. Several features are immediately apparent; first, the out-of-plane responses show more significant variability in the amplitude of the first peak compared to the in-plane response. Further, the time of the consecutive zero-crossing changes in a less regular manner for the out-of-plane displacements, as compared to the in-plane response. Undoubtedly, these differences correspond to the observed quality of related GIUM images, and we will now discuss them more in-depth, supported by analytical modelling.

To simplify the problem even more, we first asked the question - does anisotropy play a role in this phenomenon, or can it also be observed in heterogeneous, but locally isotropic material? A simple test consisted of building an 'isotropic equivalent' model, where the properties of each layer were calculated based 
a)

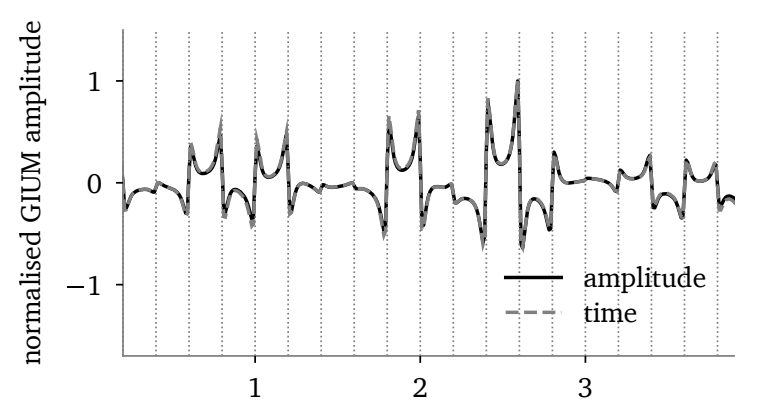

location along the vertical axis of the model in $\mathrm{mm}$ b)

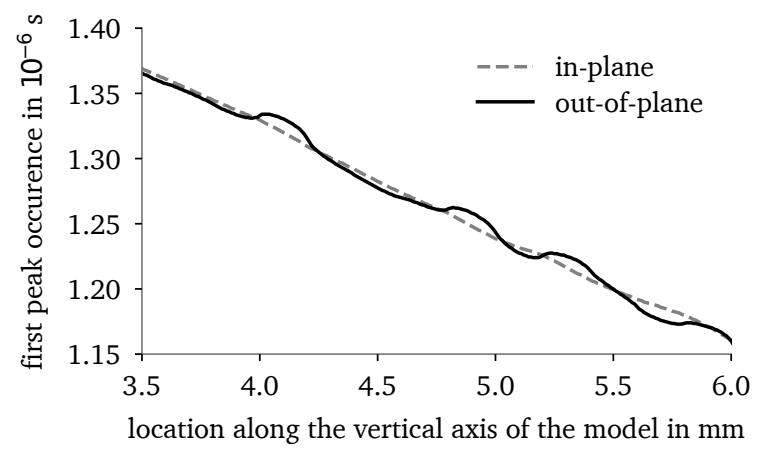

Figure 15: Equivalent locally isotropic model: a) GIUM amplitude and time traces; b) the time of the first peak for both displacement components recorded at the imaging surface; for convenience, only a proportion of the surface are shown.

on the wavespeeds in the original model at $10^{\circ}$ incidence (measured from the surface). Such a definition, while not strictly analogous, allowed for representing the local variation in properties. In Figs 14k-d we plot the displacement time traces predicted from the equivalent isotropic model across the same positions as in Figs $14 a-b$. The same features, namely the more significant variation in the amplitude of the first peak and the time of the subsequent zero-crossing, are present in both figures, confirming that the effects governing GIUM images do not require phenomena often associated with local anisotropy. The variation of the elastic properties in the direction of wave propagation on its own is sufficient, regardless of whether the medium is locally isotropic or locally anisotropic. As a final confirmation, Fig. 15a shows GIUM traces calculated for the locally isotropic model. Layer boundaries are clearly marked by sharp peaks, as in the images from the anisotropic model presented above.

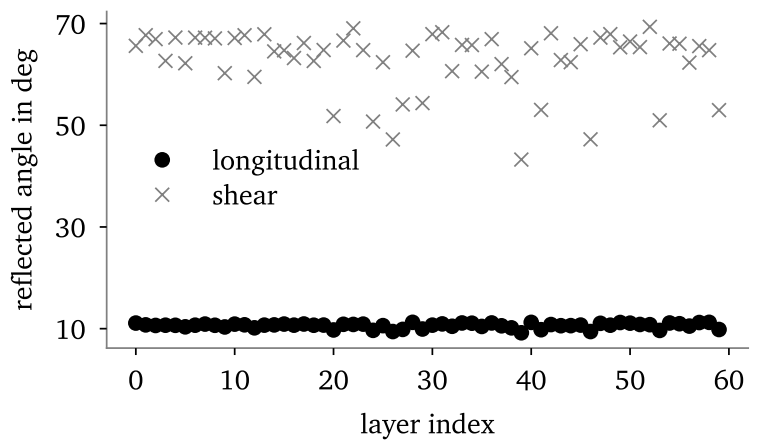

Figure 16: Analytical calculation of reflected angles at the free surface of the equivalent locally isotropic model.

43 res node

The oblique incidence calculation in the preceding subsection confirmed the relevance of the plane wave reflection perspective and allowed for conducting the analytical analysis. Considering the equivalent isotropic model, we calculate wave reflection from the free surface for each layer, accounting for the variation of the angle of incidence in the original counterpart. The analytical modelling (layer-by-layer reflection from the 

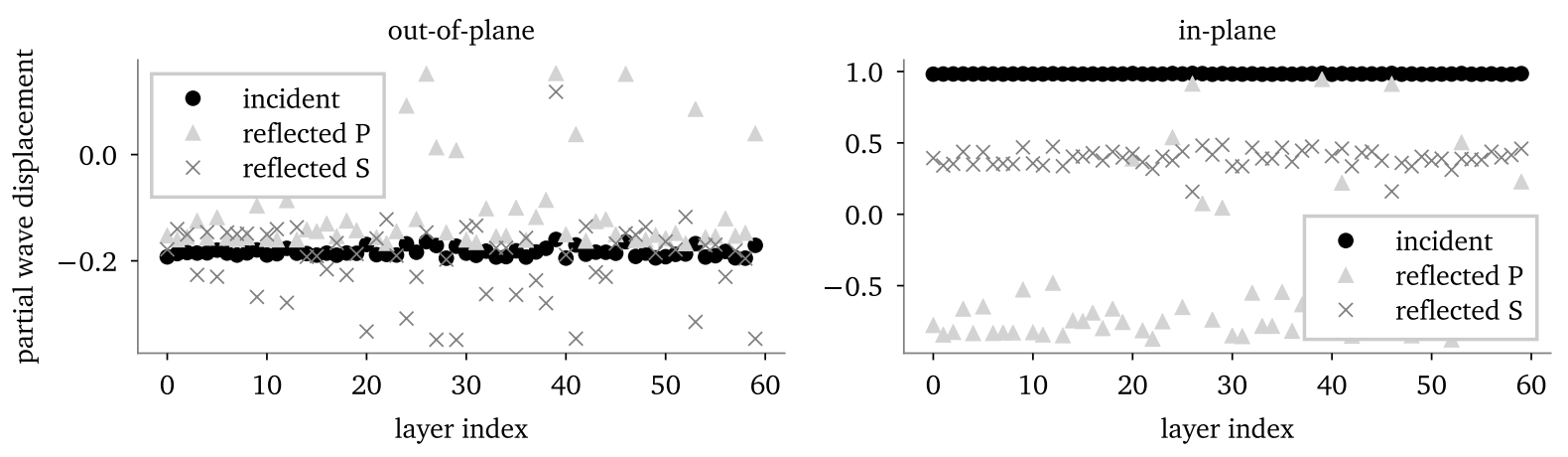

Figure 17: Analytical calculation of contributions of particular waves to the total displacement at the free surface of the equivalent locally isotropic model.

free surface) ignores any interaction between the layers, which in this case (as in reality) are much thinner than the wavelength; here wavelength/thickness $=14)$. Further, it refers to the steady-state scenario, while both in the experiment and the numerical simulations, we are analysing transient waves. Hence, the analytical calculation does not correspond to the numerical simulation directly, but we believe that it can provide an insightful perspective for analysing the results.

Figure 16 depicts the reflection angles for both $\mathrm{P}$ and $\mathrm{S}$ waves across the layers. As expected, the shear wave presents a more significant variation of angles as it is more sensitive to local changes in properties. Of most interest are the contributions of each partial wave to the total displacement at the surface, which, indirectly, can be linked to transient wave amplitudes observed. Figure 17 shows displacement components linked to each of the partial waves which, when superimposed, yield the total steady-state displacement. Let us first look at the in-plane components. The in-plane displacement originating from the incident wave dominates the response. Considering the sign difference between the in-plane displacements of the reflected waves, their contribution to the total response is several times smaller than the incident wave.

On the contrary, the out-of-plane component is dominated by both reflected waves and the variations of the associated displacement amplitudes translate into a more significant variation of the total out-of-plane displacement. The effect of local variations of properties is best pronounced in the components linked to the reflected waves and the out-of-plane component of the incident wave. This observation alludes to the behaviour of the time traces in Fig. 14. The spatial variation of the amplitude of the out-of-plane component was considerably larger than that of the in-plane counterpart. This analysis indirectly confirms that both reflected $\mathrm{P}$ and $\mathrm{S}$ waves govern the out-of-plane displacement's sensitivity to local property changes, as they dominate the out-of-plane component at the surface. The free surface facilitating wave reflection, which exposes the effect of local elastic tensor variation in the out-of-plane displacement, is the fundamental physical condition necessary for GIUM imaging to work.

Most images in this paper are calculated using the zero-crossing-based GIUM routine. We now turn our 
attention to the zero-crossing time variation. Since the layers are isotropic, the reflected $\mathrm{P}$ wave velocity is equal to the incident one, but the reflected $\mathrm{S}$ wave velocity varies significantly, along with the wavefront normal orientation. While in the steady-state the phases of all waves are perfectly matched at the free surface, in the transient scenario the phase-matching condition is underdeveloped. Hence, we expect the shear wave vector to have a notable influence on the first zero-crossing (or, equivalently, the time when the first peak occurs), given its significant contribution to the out-of-plane response. Figure $15 \mathrm{~b}$ shows the time of the first zero-crossing for both displacement components recorded at the imaging surface of the locally isotropic layered model. The variation of the zero-crossing in the in-plane displacement component is considerably smaller, as it is primarily governed by the incident wave. On the other hand, fluctuations around that line of the out-of-plane displacement zero-crossing confirm that the sensitive reflected waves contribute significantly. The 'disturbance' of the time of the first zero-crossing is small, yet large enough to be captured by the high-pass filtering used in the GIUM time image. We suggest that the transient nature of the experiment plays a key role, allowing the wavespeed variations to manifest.

GIUM amplitude imaging captures small differences in the displacement amplitude close to the zerocrossing. The analytical calculation of partial wave contributions presented above seems to refer more directly to peak amplitudes. However, we suggest that in the vicinity of the first zero-crossing the reflected waves will be better pronounced. In GIUM processing, the amplitudes are read at the low-pass filtered time instants, i.e. corresponding to the 'average' wavespeed, which follows the incident wave. As mentioned above, the phases of the partial waves do not match perfectly at the surface in a transient scenario. GIUM follows the zero-crossing of the 'average' wavefront, dominated by the incident wave. Hence, we expect that the contribution of the incident to the out-of-plane displacement is even smaller at those zero-crossings, allowing the GIUM procedure to extract information from the reflected waves, more sensitive to local property changes.

In summary, we showed that neither grazing incidence, nor local anisotropy is necessary for GIUM to map the microstructure. GIUM imaging is possible thanks to the wave reflection phenomenon at the imaging surface, which allows the more sensitive reflected partial waves to govern the out-of-plane response. Grazing incidence is an experimental convenience, but locally, given the second-order effects, the governing phenomenon is a classical reflection at the free surface with a near-grazing angle. Moreover, the transient nature of the method results in underdeveloped phase matching between the partial waves, which is exploited in the GIUM processing, enabling the local wavespeed variations to be captured.

\section{Assessing penetration depth (3D)}

3D simulations were built under the assumption that GIUM images are only affected by the grains in the vicinity of the surface. In this section, we verify this assumption, also answering a question of practical relevance to the imaging method itself - is it a surface imaging method only? 


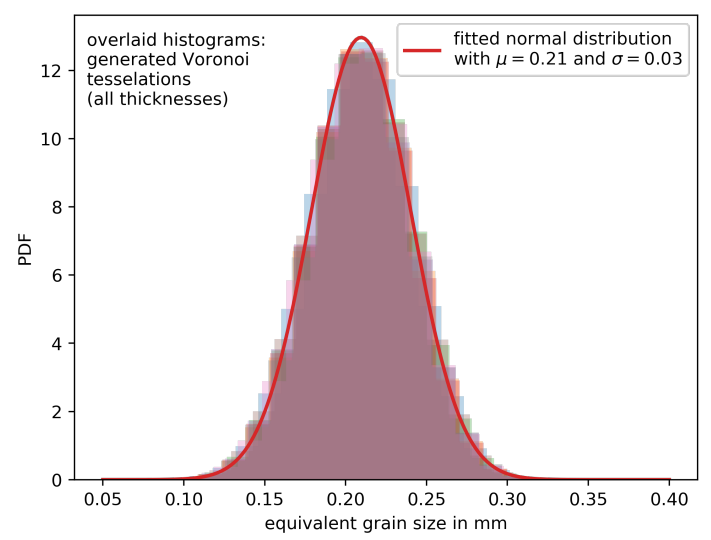

surface layer background microstructure (here $0.6 \mathrm{~mm}$ ) (Voronoi grains)

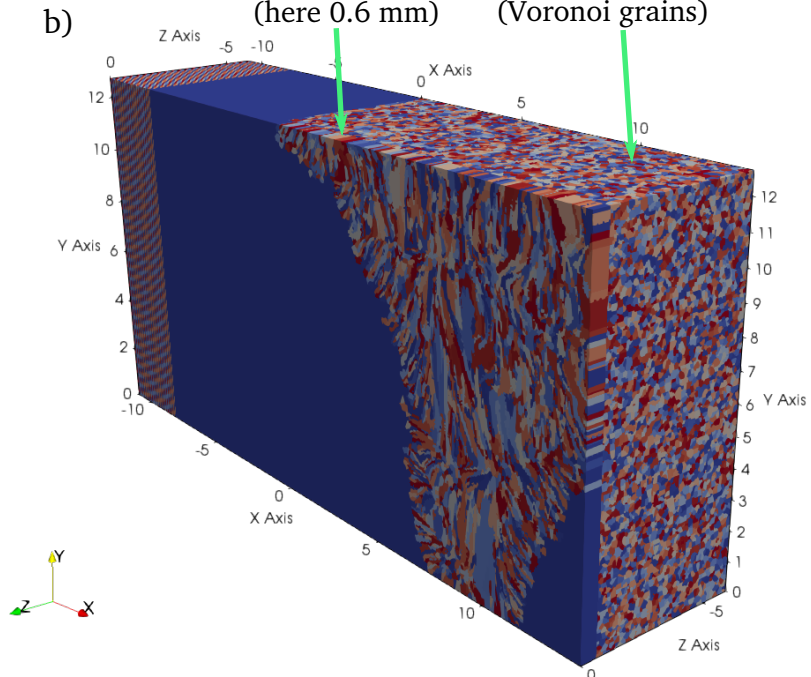

Figure 18: Penetration depth study setup: a) histograms of equivalent grain size diameters for the background layer; b) the cross-section of the FE model showing the arrangement of sections; all axis values are in mm.

In the previous section we confirmed that the detail available in GIUM images is primarily governed by the free surface reflection and the transient nature of the method. Intuitively, we expect that waves reflected from the free surface would travel into the component and reflect from the encountered grain boundaries creating structural noise. To illustrate this, we placed the extruded near-surface layer, with the microstructure synthesised based on EBSD measurements, on top of a heterogeneous background made of numerically generated grains. Changing the ratio between the thicknesses of the surface layer and the background (with the total thickness kept constant) allowed us to investigate the effect of features located at different distances from the surface on the GIUM image.

\subsection{Description}

The model was composed of two parts. The heterogeneous background was a Voronoi tessellation with approximately normally distributed grain sizes. The size of background grains was comparable to the 'thickness' of the columnar grains in the 2D EBSD image. The histograms of equivalent grain diameters of the background layers (all are nearly identical) and the fitted normal distribution are shown in Fig. 18 a.

The crystallographic orientations in the background were random, yielding a macroscopically isotropic material. We used the same crystal stiffness matrix for both the surface layer and the background (see Eq. 1). Eight thicknesses of the surface layer were considered: $0.05,0.1,0.2,0.4,0.6,0.8,1$, and $2 \mathrm{~mm}$. The thickness of the background was adjusted accordingly to achieve a total thickness of $6 \mathrm{~mm}$ (or 120 elements, given the $0.05 \mathrm{~mm}$ element size). The absorbing layers and the excitation were the same as in the 3D model described above. The main difference was that to the left and to the right of the weld, the material was defined as isotropic in the surface layer, but it was kept heterogeneous for the background. This arrangement 
allowed for gaining a clearer insight into the effect of the background microstructure on the GIUM image.

A cross-section of the model is shown in Fig. $18 \mathrm{~b}$.

This model had the same number of degrees of freedom as that in Section 4, but at the same time, it had a more complex material structure. It was solved using Pogo on a cluster node with 8 Nvidia RTX 8000 GPU cards. It is worth noting that the capability of this system well exceeds 1 billion degrees of freedom with Pogo. However, it was used for this model to make sure that the memory requirements dictated by the very large number of materials were met comfortably.

\subsection{Results}

The amplitude GIUM images for all the chosen configurations are presented in Fig. 19 . The comparison indicates that features below the surface affect the surface image only to a very small depth, comparable to the minor axis length of the columnar grains. Even for the thinnest surface layer of $0.05 \mathrm{~mm}$, the columnar grains pattern is still discernible. Above $0.2 \mathrm{~mm}$ the distortion to the weld area is merely noticeable, whereas above $0.4 \mathrm{~mm}$, one could hardly distinguish any grains through the isotropic regions outside of the weld.

To illustrate these observations more clearly, we plotted two horizontal cross-sections through the images, one through the line $y=2.5 \mathrm{~mm}$ and the other through $y=8.5 \mathrm{~mm}$. The line plots are shown in Fig. 20

This study allows us to conclude that GIUM is a surface imaging method, and features deeper below the surface than a typical feature size (here, it is the thickness of the columnar grain) neither can be distinguished, nor do they notably affect the generated images. Furthermore, signatures of grains at the imaging surface are always strongly dominant, even above a closely placed heterogeneous background.

\section{Conclusions}

\section{Acknowledgement}

The first two authors were supported by the ADVISE project (www.advise-h2020.eu) funded from the Euratom research and training programme 2014-2018 under grant agreement no. 755500.

\section{References}

[1] A. J. Schwartz, Electron backscatter diffraction in materials science, Springer, 2009.

[2] C. Ilett, M. Somekh, G. Briggs, Acoustic microscopy of elastic discontinuities, Proceedings of the Royal Society of London. A. Mathematical and Physical Sciences 393 (1984) 171-183. Publisher: The Royal Society London.

[3] J.-I. Kushibiki, M. Arakawa, A method for calibrating the line-focus-beam acoustic microscopy system, IEEE transactions on ultrasonics, ferroelectrics, and frequency control 45 (1998) 421-430. Publisher: IEEE.

[4] S. Sathish, M. Mendik, J. H. Cantrell, W. T. Yost, Elastic constants and crystal orientation of individual grains of polycrystalline solids from scanning acoustic microscopy, The Journal of the Acoustical Society of America 98 (1995) 2854-2857. Publisher: Acoustical Society of America.

[5] U. Rabe, W. Arnold, Acoustic microscopy by atomic force microscopy, Applied Physics Letters 64 (1994) $1493-1495$. Publisher: American Institute of Physics.

[6] U. Rabe, M. Kopycinska, S. Hirsekorn, J. M. Saldana, G. Schneider, W. Arnold, High-resolution characterization of piezoelectric ceramics by ultrasonic scanning force microscopy techniques, Journal of Physics D: Applied Physics 35 (2002) 2621. Publisher: IOP Publishing.

[7] M. Kopycinska, C. Ziebert, H. Schmitt, U. Rabe, S. Hirsekorn, W. Arnold, Nanoscale imaging of elastic and piezoelectric properties of nanocrystalline lead calcium titanate, Surface science 532 (2003) 450-455. Publisher: Elsevier. 
[8] S. D. Sharples, M. Clark, M. G. Somekh, Spatially resolved acoustic spectroscopy for fast noncontact imaging of material microstructure, Optics express 14 (2006) 10435-10440. Publisher: Optical Society of America.

[9] S. D. Sharples, M. Clark, W. Li, M. G. Somekh, Rapid imaging of microstructure using spatially resolved acoustic spectroscopy, in: 1st International Symposium on laser Ultrasonics Montreal, 2008.

[10] A. Mark, W. Li, S. Sharples, P. Withers, Comparison of grain to grain orientation and stiffness mapping by spatially resolved acoustic spectroscopy and EBSD, Journal of microscopy 267 (2017) 89-97. Publisher: Wiley Online Library.

[11] M. Clark, A. Clare, P. Dryburgh, W. Li, R. Patel, D. Pieris, S. Sharples, R. Smith, Spatially resolved acoustic spectroscopy (SRAS) microstructural imaging, in: AIP Conference Proceedings, volume 2102, AIP Publishing LLC, 2019 , p. 020001. Issue: 1 .

[12] B. Köhler, M. Kehlenbach, R. Bilgram, Optical measurement and visualization of transient ultrasonic wave fields, in: Acoustical Imaging, Springer, 2004, pp. 315-322.

[13] B. Köhler, Scanning acquisition of ultrasonic wave fields for non-destructive testing, non-destructive material characterization and structural health monitoring: habilitation thesis, PhD Thesis, habilitation thesis, Technische Universität Dresden, 2014.

[14] K. Nakahata, H. Sugahara, M. Barth, B. Köhler, F. Schubert, Three dimensional image-based simulation of ultrasonic wave propagation in polycrystalline metal using phase-field modeling, Ultrasonics 67 (2016) 18-29. URL: http://www. sciencedirect.com/science/article/pii/S0041624X15003194 doi 10.1016/j.ultras.2015.12.013

[15] U. Völz, H. Mrasek, K. Matthies, H. Wüstenberg, M. Kreutzbruck, Electrodynamic Approach for Visualization of Sound Propagation in Solids, in: AIP Conference Proceedings, volume 1096, 2009, pp. 758-765.

[16] M. Barth, Control device for a stimulation signal generator, German patent: DE102012215856A1, International patent: WO2014037387A1.

[17] B. Köhler, M. Barth, P. Krüger, F. Schubert, Grain structure visualization with surface skimming ultrasonic waves detected by laser vibrometry, Applied Physics Letters 101 (2012) 074101-074101. Publisher: AIP.

[18] B. Kohler, M. Barth, F. Schubert, Experimental study of ultrasonic wave propagation for austenitic and dissimilar welds, in: Ultrasonics Symposium (IUS), 2012 IEEE International, IEEE, 2012, pp. 261-264.

[19] B. Köhler, M. Barth, F. Schubert, A new application of laser vibrometric wave field propagation measurements: Grain structure visualization, in: AIP Conference Proceedings, volume 1511, 2013, pp. 1059-1064

[20] M. Barth, B. Köhler, F. Schubert, Grain Structure Visualization of Austenitic Welds by Laser Detection of Grazing Incident Ultrasonic Wave Fields, in: Proceedings of the International Symposium of Nondestructive Characterization of Materials, May 2014, Le Mans, France, www.ndt.net/?id=15534, 2014.

[21] P. Huthwaite, Accelerated finite element elastodynamic simulations using the GPU, Journal of Computational Physics 257 (2014) 687-707. URL: http://www.sciencedirect.com/science/article/pii/S0021999113006931. doi 10.1016/j. jcp.2013.10.017

[22] A. V. Pamel, G. Sha, S. I. Rokhlin, M. J. S. Lowe, Finite-element modelling of elastic wave propagation and scattering within heterogeneous media, Proc. R. Soc. A 473 (2017) 20160738. URL: http://rspa.royalsocietypublishing.org/ content/473/2197/20160738 doi $10.1098 /$ rspa.2016.0738

[23] A. Van Pamel, G. Sha, M. J. S. Lowe, S. I. Rokhlin, Numerical and analytic modelling of elastodynamic scattering within polycrystalline materials, The Journal of the Acoustical Society of America 143 (2018) 2394-2408. URL: https: //asa.scitation.org/doi/full/10.1121/1.5031008 doi 10.1121/1.5031008 publisher: Acoustical Society of America.

[24] X. Bai, B. Tie, J. H. Schmitt, D. Aubry, Finite element modeling of grain size effects on the ultrasonic microstructural noise backscattering in polycrystalline materials, Ultrasonics 87 (2018) 182-202. URL: http://www.sciencedirect.com/ science/article/pii/S0041624X18301069 doi 10.1016/j.ultras.2018.02.008.

[25] X. Bai, B. Tie, J. H. Schmitt, D. Aubry, Comparison of ultrasonic attenuation within two- and three-dimensional polycrystalline media, Ultrasonics 100 (2020) 105980. URL: http://www.sciencedirect.com/science/article/pii/ S0041624X18308096 doi 10.1016/j.ultras.2019.105980

[26] M. Ryzy, T. Grabec, P. Sedlák, I. A. Veres, Influence of grain morphology on ultrasonic wave attenuation in polycrystalline media with statistically equiaxed grains, The Journal of the Acoustical Society of America 143 (2018) 219-229. URL: https://asa.scitation.org/doi/10.1121/1.5020785 doi 10.1121/1.5020785, publisher: Acoustical Society of America.

[27] Y. Liu, A. Van Pamel, P. B. Nagy, P. Cawley, Investigation of ultrasonic backscatter using three-dimensional finite element simulations, The Journal of the Acoustical Society of America 145 (2019) 1584-1595. URL: https://asa.scitation.org/ doi/full/10.1121/1.5094783 doi 10.1121/1.5094783 publisher: Acoustical Society of America.

[28] B. Chassignole, V. Duwig, M. A. Ploix, P. Guy, R. El Guerjouma, Modelling the attenuation in the ATHENA finite elements code for the ultrasonic testing of austenitic stainless steel welds, Ultrasonics 49 (2009) 653-658. URL: http: //www.sciencedirect.com/science/article/pii/S0041624X0900047X doi 10.1016/j.ultras.2009.04.001

[29] P. E. Lhuillier, B. Chassignole, M. Oudaa, S. O. Kerhervé, F. Rupin, T. Fouquet, Investigation of the ultrasonic attenuation in anisotropic weld materials with finite element modeling and grain-scale material description, Ultrasonics 78 (2017) $40-50$. URL: http://www.sciencedirect.com/science/article/pii/S0041624X17302184 doi 10.1016/j.ultras.2017.03.004

[30] Y. Chen, Z. Luo, Q. Zhou, L. Zou, L. Lin, Modeling of ultrasonic propagation in heavy-walled centrifugally cast austenitic Stainless steel based on EBSD analysis, Ultrasonics 59 (2015) 31-39. URL: http://www.sciencedirect.com/science/ article/pii/S0041624X15000116 doi 10.1016/j.ultras.2015.01.009

[31] S. Wagner, S. Dugan, M. Barth, F. Schubert, B. Köhler, Welding for testability: An approach aimed at improving the ultrasonic testing of thick-walled austenitic and dissimilar metal welds, in: 40th Annual Review Of Progress In Quantitative Nondestructive Evaluation: Incorporating the 10th International Conference on Barkhausen Noise and Micromagnetic Testing, volume 1581, AIP Publishing, 2014, pp. 1045-1052.

[32] M. A. Groeber, M. A. Jackson, DREAM.3D: A Digital Representation Environment for the Analysis of Microstructure in 
3D, Integrating Materials and Manufacturing Innovation 3 (2014) 56-72. URL: https://doi.org/10.1186/2193-9772-3-5 doi $10.1186 / 2193-9772-3-5$

631 [33] H. Ledbetter, Monocrystal elastic constants in the ultrasonic study of welds, Ultrasonics 23 (1985) 9

- 13. URL: http://www.sciencedirect.com/science/article/pii/0041624X85900058 doi https://doi.org/10.1016/ 0041-624X(85) 90005-8

634 [34] A. van Pamel, C. R. Brett, P. Huthwaite, M. J. S. Lowe, Finite element modelling of elastic wave scattering within a polycrystalline material in two and three dimensions, The Journal of the Acoustical Society of America 138 (2015) 2326-2336. URL: http://asa.scitation.org/doi/abs/10.1121/1.4931445 doi 10.1121/1.4931445

37 [35] www.pogo.software, Pogo - high speed wave simulations, 2020. URL: http://www.pogo.software/

638 [36] T. E. Oliphant, A guide to NumPy, Trelgol Publishing, USA, 2006.

639 [37] P. A. Heelan, On the theory of head waves, Geophysics 18 (1953) 871-893. URL: http://library.seg.org/doi/10.1190/ 1.1437941 doi $10.1190 / 1.1437941$ 

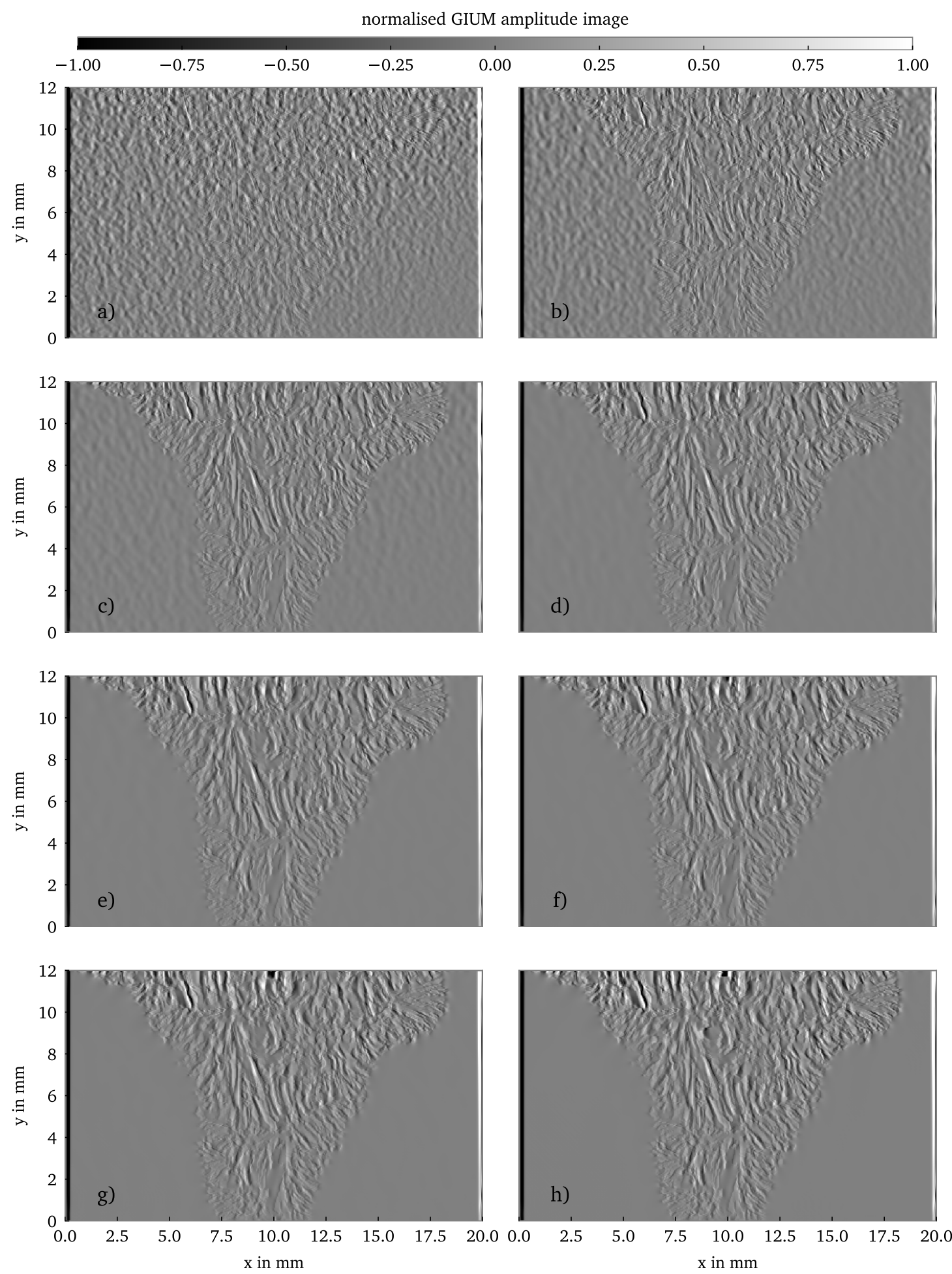

Figure 19: Normalised GIUM 'zero-crossing' amplitude images generated for different thicknesses of the surface layer, with the total thickness of the model kept constant at $6 \mathrm{~mm}$ : a) $0.05 \mathrm{~mm}$, b) $0.1 \mathrm{~mm}$, c) $0.2 \mathrm{~mm}$, d) $0.4 \mathrm{~mm}$, e) $0.6 \mathrm{~mm}, \mathrm{f}) 0.8 \mathrm{~mm}, \mathrm{~g}$ ) $1 \mathrm{~mm}, \mathrm{~h}) 2 \mathrm{~mm}$ 
a)

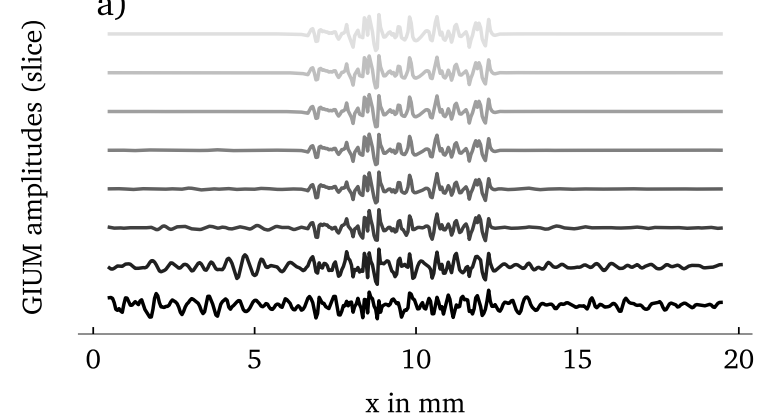

b)

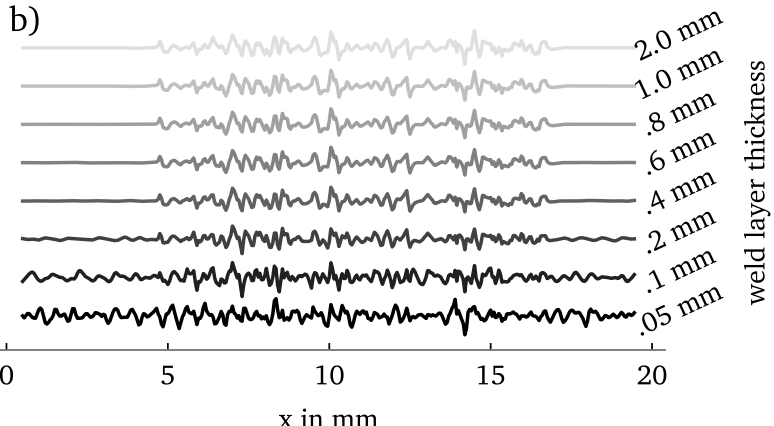

Figure 20: Cross-sections through penetration depth study images for different thicknesses of the surface layer (denoted on the right vertical axis): a) at $y=2.5 \mathrm{~mm}, \mathrm{~b}$ ) at $y=8.5 \mathrm{~mm}$. 